\title{
BacPROTACs mediate targeted protein degradation in bacteria
}

Francesca Ester Morreale ${ }^{1}$, Stefan Kleine ${ }^{2}$, Julia Leodolter ${ }^{1}$, Stepan Ovchinnikov ${ }^{1}$, Juliane Kley ${ }^{1}$, Robert Kurzbauer ${ }^{1}$, David M. Hoi ${ }^{1}$, Anton Meinhart ${ }^{1}$, Markus Hartl ${ }^{3}$,

5 David Haselbach ${ }^{1}$, Markus Kaiser ${ }^{2}$, Tim Clausen ${ }^{1,4, *}$

${ }^{1}$ Research Institute of Molecular Pathology, Vienna BioCenter, Vienna, Austria

2 University of Duisburg-Essen, Center of Medical Biotechnology, Faculty of Biology,

10 Essen, Germany

${ }^{3}$ Max Perutz Labs, Vienna BioCenter, Vienna, Austria

${ }^{4}$ Medical University of Vienna, Vienna, Austria

*Correspondence should be addressed to Tim Clausen, tim.clausen@imp.ac.at 


\section{Summary}

Hijacking the cellular protein degradation system offers unique opportunities for drug discovery, as exemplified by proteolysis targeting chimeras (PROTACs). Despite their superior properties over classical inhibitors, it has so far not been possible to reprogram the bacterial degradation machinery to interfere with microbial infections. Here, we develop small-molecule degraders, so-called BacPROTACs, that bind to the substrate receptor of the ClpC:ClpP protease, priming neo-substrates for degradation.

30 In addition to their targeting function, BacPROTACs activate $\mathrm{ClpC}$, transforming the resting unfoldase into its functional state. The induced higher-order oligomer was visualized by cryo-EM analysis, providing a structural snapshot of activated ClpC unfolding a protein substrate. Finally, degradation assays performed in mycobacteria demonstrate in vivo activity of BacPROTACs, highlighting the potential of the 35 technology to provide next generation antibiotics. 


\section{INTRODUCTION}

Technological advances in proteomics, chemical biology and high throughput screening campaigns have boosted drug discovery across therapeutic areas. Unfortunately, however, these advances have thus far not equally translated into the development of novel antibacterial agents (Payne et al., 2007; Tommasi et al., 2015). This discrepancy is most obvious when considering the small number of antibiotics that have been discovered in the last 50 years (Lewis, 2020). Beyond economic

45 hurdles, the advancement of new antibiotics is challenged by the low permeability of the bacterial envelope, and the limited number of microbial proteins that can be specifically inhibited without off-target effects. The difficulties in finding effective antimicrobials are further compounded by the speed at which pathogens are developing resistance to existing drugs. In light of this unequal arms race, the return of bacterial

50 pandemics is a real threat, and innovative strategies to combat infections by multiresistant bacteria are urgently needed (Lewis, 2020).

An emerging concept in drug discovery is the induced elimination of target proteins. Engineered chemicals can now interfere with various degradation pathways, redirecting the lysosomal (Banik et al., 2020), the autophagy (Li et al., 2019; Takahashi et al., 2019) or the ubiquitin-proteasome systems (Sakamoto et al., 2001) to target specified proteins. The most prominent synthetic "degraders" are the Proteolysis Targeting Chimeras (PROTACs): bi-functional small molecules that contain a binding head for an E3 ubiquitin ligase and a chemical moiety to engage a protein of interest (POI) (Sakamoto et al., 2001). By bringing the E3 ligase and POI into proximity,

60 PROTACs promote POI ubiquitination and consequent degradation by the proteasome. Protein degraders have various advantages over classical inhibitors. For example, they exhibit higher efficacy due to their catalytic mode of action. Moreover, they allow targeting of virtually any cellular protein and their modular architecture allows protein ligands to be repurposed to build degraders (Churcher, 2018; Hanzl and

65 Winter, 2020; Schapira et al., 2019). Despite this promise, PROTAC technology is so far restricted to the ubiquitin tagging system of eukaryotes and has yet to be transferred to degradation pathways in bacteria. Fulfilling this last objective would be a powerful addition to the antibiotic arsenal. 
Although ubiquitin is unique to eukaryotic cells, some bacteria utilize a similar system

for targeted protein degradation. Phosphorylated arginine residues (pArg) serve as a degradation signal that is recognized by the ClpC:ClpP (ClpCP) protease, a quasiproteasomal particle critical for microbial protein quality control, stress tolerance and pathogenicity. CIpCP is present in Gram-positive bacteria and in mycobacteria. In the latter, the equivalent CIpC1P1P2 protease is essential for survival in vitro and in macrophages (DeJesus et al., 2017; Rengarajan et al., 2005). ClpC carries out its quality control function by acting as an ATP-driven unfoldase that selects certain client proteins and translocates them into the protease compartment formed by ClpP. The ClpC protomer contains an amino-terminal domain (ClpC $\mathrm{NTD}_{\mathrm{N}}$ ), two AAA (ATPases Associated with diverse cellular Activities) domains termed D1 and D2, and a coiledcoil (M-domain) inserted into D1. In the active ClpC hexamer, the D1 and D2 domains form two stacked ATPase rings that power substrate unfolding and translocation. The $\mathrm{ClpC}_{\mathrm{NTD}}$ on top of the D1 ring controls access to the unfoldase, providing docking sites for adaptor proteins including MecA (Schlothauer et al., 2003; Wang et al., 2011). In addition, the ClpCNTD receptor domain recognizes pArg-tagged substrates that account for $\sim 30 \%$ of the whole ClpP degradome in Gram-positive bacteria (Trentini et al., 2016). Compared to the eukaryotic proteasome, which recognizes a complex polyubiquitin signal, the degradation signal recognized by CIpCP is much simpler: a plain phosphate group attached to an arginine residue of the client protein (Trentini et al., 2016). Here we investigated whether the ClpCP degradation machine can be directly reprogrammed by small molecules. We reasoned that pArg-containing chemical adaptors could tether neo-substrates to the $\mathrm{ClpC}_{\mathrm{NTD}}$ receptor domain, priming them for degradation (Figure 1A). Our results provide a proof of concept that such BacPROTACs can be developed and are active in vivo, enabling the inducible, selective and efficient degradation of target proteins in bacteria. 


\section{RESULTS}

\section{Development of BacPROTACs that reprogram CIpCP}

To enable ClpC-mediated protein degradation, we designed BacPROTACs composed 100 of a POI ligand, a chemical linker and a CIPC $C_{N T D}$ anchor. The anchor initially consisted of a peptidic pArg derivative mimicking the bacterial degradation tag. In order to test the targeting of a neo-substrate, we used monomeric streptavidin (mSA) as a model protein. We synthesized BacPROTAC-1 (Figure 1B), connecting the pArg moiety to biotin, a high affinity mSA ligand (Lim et al., 2013). The linker attachment points were

105 designed based on high-resolution crystal structures of pArg:ClpCNTD (Trentini et al., 2016) and biotin:mSA (Demonte et al., 2013). Isothermal titration calorimetry (ITC) measurements confirmed that BacPROTAC-1 binds mSA and CIpCNTD with high affinity (KDS of $3.9 \mu \mathrm{M}$ and $2.8 \mu \mathrm{M}$ Figure 1C and 1D), whereas analytical size exclusion chromatography (SEC) runs revealed the formation of a stable ternary

110 complex (Figure 1E). To analyze whether the induced spatial proximity is sufficient to trigger degradation, we reconstituted the Bacillus subtilis ClpCP protease in vitro and monitored mSA digestion at different BacPROTAC concentrations. Incubation with $100 \mu \mathrm{M}$ BacPROTAC-1 led to selective mSA depletion (Figure 1F), indicating that the bacterial ClpCP can be reprogrammed by a pArg-containing chemical adaptor.

115 Notably, however, efficient degradation was only observed at concentrations that were higher than the measured affinities among individual components. Presumably, this discrepancy reflects distinct requirements for substrate recruitment and translocation processes carried out by the ClpC unfoldase.

To follow up on this and analyze the influence of substrate-specific properties on

120 ClpCP activity, we cloned various mSA fusion proteins. We selected four $B$. subtilis targets that have been identified as physiological, pArg-labelled ClpCP substrates (Trentini et al., 2016). Three of these proteins (Nrdl, TagD, NusA) adopt compact protein folds, whereas Kre is predicted to be a mostly unstructured protein (Figure S1). In vitro assays, monitoring the BacPROTAC-1 dependent degradation of 125 the four mSA fusion proteins by ClpCP, revealed pronounced differences (Figure 1G and $\mathbf{1 H}$ ). mSA-Kre was by far the best substrate, being degraded by $1 \mu \mathrm{M}$ BacPROTAC. These in vitro data suggest that ClpCP preferentially acts on proteins with unstructured regions, which could serve as initiator sites for substrate unfolding 
130265 proteasome, which requires an unstructured region to efficiently degrade ubiquitinated proteins (Inobe et al., 2011; Prakash et al., 2004).

To confirm that degradation is specifically induced by BacPROTAC-1, we hindered substrate recruitment by adding the isolated binding moieties pArg or biotin to the reaction (Figure $\mathbf{1 H}$ ). In the presence of these compounds, mSA-Kre was not digested

135 by ClpCP, confirming that BacPROTAC-mediated ternary complex formation is essential for degradation. Taken together, our results show that pArg-containing BacPROTACs can recruit POIs to the CIPCNTD domain and promote their degradation by the ClpCP protease. In addition to the binding characteristics of the chemical adaptor, intrinsic properties of target proteins seem to play an equally important role

140 in determining degradation efficiencies.

\section{BacPROTAC binding induces ClpC reassembly and activation}

Housekeeping proteases and chaperones that target aberrant proteins need to be carefully controlled to prevent concomitant damage to functional proteins in the cell. A study addressing the regulation of the Staphylococcus aureus $\mathrm{ClpC}$, a close relative

145 to the $B$. subtilis unfoldase, revealed the existence of a ClpC resting state, a decamer with disrupted AAA rings (Carroni et al., 2017). Interaction with the adaptor protein MecA destabilizes the decamer and promotes assembly of the functional hexamer with an active arrangement of ATPase units (Carroni et al., 2017). In contrast to adaptor-mediated activation, it is unclear how the majority of $\mathrm{ClpC}$ substrates labelled

150 with pArg trigger remodeling of the latent $\mathrm{ClpC}$ decamer. To elucidate this general activation mechanism - and more specifically, the way in which it is mimicked by a pArg-based degrader - we performed a structural analysis of $\mathrm{ClpC}$ in complex with BacPROTAC-1 and the mSA-Kre fusion protein. To stabilize ATP-mediated contacts between CIpC protomers, we used a catalytically inactive mutant (E280A/E618A,

155 Clp mSA-Kre in stoichiometric amounts to a SEC column, ClpCDWB and mSA-Kre eluted separately (Figure 2A). Like the $S$. aureus protein, isolated B. subtilis ClpCDwB was present in its resting state, the decamer, as visualized by negative-staining EM (Figure 2B and S2A). Incubation with BacPROTAC-1 led to the co-elution of mSA and 160 ClpCDwB, pointing to the formation of a stable ternary complex. To our surprise, 
however, the estimated size of the BacPROTAC induced complex was far from compatible with the predicted size of substrate-bound ClpC $C_{\text {Dw }}$ hexamer (Figure 2A). Instead the resulting complex behaved like a higher-order $\mathrm{ClpC}$ oligomer with a molecular mass beyond 2 MDa. Cryo-EM analysis of the substrate-engaged ClpC revealed the formation of a tetramer of ClpC hexamers, arranged in almost perfect tetrahedral symmetry (Figure 2C and S2B). A similar arrangement has been reported for a chimeric CIpC (M. tuberculosis NTD fused to $S$. aureus D1-D2) incubated with the antibiotic cyclomarin A (CymA) (Maurer et al., 2019). However, in that case, 2D class averages of negative-stained EM images could not reveal structural details of the tetrahedral assembly. BacPROTAC-tethered mSA-Kre, which mimics a trapped substrate, yielded seemingly better-defined particles of the activated state. Cryo-EM analysis visualized the overall organization of the higher-order ClpC unfoldase complex at $10 \AA$ resolution (Figure 2D and S2C, Table 1). In this state, the D2 rings of the four ClpC hexamers project outwards such that they can interact with the CIpP

175 protease. The substrate-bound $\mathrm{CIPC}_{\text {NTD }}$ domains are located in the center of the particle but are too flexible to be defined by EM density. Most strikingly, the four CIpC hexamers interact with each other via their coiled-coil M-domains, establishing a net of helix pairs holding the particle together (Figure 2D). As the M-domains are known to stabilize the resting state of CIpC (Carroni et al., 2017), their BacPROTAC-induced 180 reorientation might disassemble the latent form and promote formation of active hexamers stabilized within a supramolecular assembly (Figure 2E). To resolve the functional units of the activated unfoldase at higher resolution, we performed a focused cryo-EM analysis of the single hexamers (Figure 3A). The resulting 3D map had an overall resolution of $3.7 \AA$ (Figure S2C-E and Table 1) and allowed us to build an atomic model of the substrate-bound ClpC complex (Figure 3, Movie S1).

The most prominent feature of the cryo-EM reconstruction is a well-defined, $80 \AA$-long density that penetrates the entire ClpC pore, proceeding from the top of the $\mathrm{D} 1$ to the bottom of the D2 ring (Figure 3B). Although it is not possible to discern side chains, the density should represent the protein backbone of the captured mSA-Kre substrate, comprising 26 residues present in an extended conformation. The six ClpC subunits adopt a spiral arrangement, engaging the substrate in a similar manner to that observed in the related double-ring AAA unfoldases ClpA, ClpB, and Hsp104 (Gates et al., 2017; Lopez et al., 2020; Rizo et al., 2019). Five ClpC protomers (P1-P5, P1 as 
lowermost and P5 uppermost unit in the AAA ring) interact with the substrate through conserved tyrosine-bearing pore loops in the D1 and D2 domains, while the "seam" protomer (P6) is detached from the substrate, transitioning from the bottom to the top position of the spiral (Figure 3C and 3D). Nucleotide states were assigned on the basis of the EM density and the position of the so-called arginine fingers from the neighboring subunit (Figure S3). Accordingly, the D1 and D2 ATPase rings act in a

200 closely coordinated manner to translocate substrate through the central pore. In both rings, the substrate-engaging P2-P5 protomers are present in an ATP-bound state (except P2-D2), whereas protomers P1 and P6 accommodate ADP or are present in an apo state. Overall, the structural data are consistent with the previously suggested "hand-over-hand" translocation mechanism (Puchades et al., 2020), according to

205 which nucleotide exchange is coupled with substrate release and upward movement of the seam protomer P6 to rebind ATP and substrate (Figure 3E). By reorienting in a concerted manner, the $\mathrm{ClpC}$ protomers move stepwise from one end of the spiral to the other, dragging the captured substrate down the central channel (Puchades et al., 2020). Cycles of substrate binding, translocation and release are driven by ATP 210 hydrolysis at the lowermost P1 protomer of the spiral. In conclusion, the cryo-EM structure of the reconstituted ternary complex provides a snapshot of $\mathrm{ClpC}$ in the process of unfolding a BacPROTAC-tethered substrate. The structure of the CIpC 24mer indicates that the pArg mark serves not only as a degradation signal, but also mediates higher-order oligomer formation and activation of $\mathrm{ClpC}$. The developed

215 BacPROTAC containing a pArg moiety triggers this remodeling mechanism and thus functions not only as a chemical adaptor, but also as an activator of the CIpCP protease.

\section{Extending the BacPROTAC approach to mycobacteria}

The above biochemical and structural data revealed that pArg-based BacPROTACs

220 can reprogram the ClpCP system of $B$. subtilis. To examine the therapeutic potential of the developed degrader, we aimed to transfer our approach to mycobacteria, which are among the most widely spread and dangerous human pathogens (World Health Organization, 2019). Although a pArg-dependent degradation pathway has not been identified in mycobacteria, the substrate receptor of the ClpC1P1P2 protease has a 225 bonafide pArg-binding site, with all $\mathrm{ClpC1NTD}$ residues implicated in binding the 
terminal phospho-guanidinium being conserved (Figure S4). To test if pArg-based BacPROTACs can reprogram the mycobacterial degradation machinery, we analyzed the Mycobacterium smegmatis Clp system. ITC and SEC experiments revealed that BacPROTAC-1 binds to CIpC1NTD with high affinity $\left(\mathrm{K}_{\mathrm{D}}=0.69 \mu \mathrm{M}\right)$ and promotes 230 ternary complex formation with mSA and CIpC1NTD (Figure 4A and S5A). We then reconstituted the CIpC1P1P2 protease in vitro and observed that BacPROTAC-1 induces the degradation of a mSA substrate in a highly selective and efficient manner (Figure 4B). These data demonstrate that pArg-containing degraders can reprogram the ClpC1P1P2 protease of mycobacteria.

235 A major limitation in advancing pArg-based PROTACs is their poor pharmacokinetic profile and the chemical instability of the phospho-guanidinium group (Schmidt et al., 2014). To overcome these limitations, we looked for chemical entities that could replace pArg. Of note, the mycobacterial $\mathrm{ClpC} 1$ is targeted by a range of cyclic peptides that deregulate the CIpC1P1P2 complex (Lee and Suh, 2016). These

240 antibiotics bind to the substrate receptor domain and thus have a pArg-like targeting function. The best characterized CIpC1NTD-directed antibiotic is CymA (Vasudevan et al., 2013), which is accommodated in a hydrophobic pocket located in a remote position relative to the pArg binding sites (Figure $4 \mathbf{C}$ ). The CymA binding pocket is highly conserved in ClpC1 unfoldases, but is absent in ClpC proteins from Gram245 positive bacteria, enabling selective targeting of the mycobacterial protease. Moreover, CymA can pass the cell envelope of mycobacteria (Schmitt et al., 2011) and thus represents a building block with important properties that could expand the potential of small molecule degraders in bacterial cells.

To prepare CymA-based degraders, we developed a solid-phase synthesis approach 250 providing the 7-residue cyclic peptide in large quantities. To facilitate the intricate de novo synthesis of the natural compound (Barbie and Kazmaier, 2016), we replaced certain non-proteinogenic amino acids with chemically simpler analogues. Guided by structural data (Figure 4D) (Vasudevan et al., 2013), we prepared a series of CymAlike cyclic peptides and identified SCym-1 as a high affinity $C / p C 1_{N T D}$ ligand $\left(K_{D}=0.81\right.$ $255 \mu \mathrm{M}$, Figure S5B). A co-crystal structure obtained at $1.7 \AA$ resolution (Figure 4E, Table S1) confirmed that the SCym-1 mimetic adopts the same binding mode as the native CymA antibiotic. Moreover, the crystal structure revealed a possible linker attachment site to synthesize BacPROTAC-2, in which sCym-1 is linked to biotin 
(Figure 4F, 4G and S5C). When tested in our in vitro assay, the sCym-1-based degrader stimulated removal of the mSA model substrate to similar extents as its pArg counterpart (Figure $\mathbf{4 H}$ ), indicating that derivatives of the CymA antibiotic can be repurposed as BacPROTAC components. Importantly, these data also show that various CIpC1 NTD binders can be exploited to develop chemical adaptors for targeted protein degradation.

\section{BacPROTAC induces protein degradation in mycobacteria}

We next analyzed whether BacPROTACs can induce POI degradation when introduced into mycobacteria. To show the system is generalizable, and since cellular biotin would compete for binding to $\mathrm{mSA}$ and impede BacPROTAC activity (Figure 4H), we looked for an alternative POI and identified the bromodomain-1 (BD1)

270 of BRDT as attractive model substrate. BRDTBD1 (residues 21-137) encodes a soluble protein that binds with high affinity to BET bromodomain inhibitors (Matzuk et al., 2012). One of its small molecule ligands, JQ1, has been widely used in various PROTACs (Winter et al., 2015; Zengerle et al., 2015), thus facilitating the rational design of the bacterial degrader. Moreover, BRDT is a human protein with no bacterial 275 homologues, allowing tracking of in vivo degradation without interfering with endogenous pathways. To target BRDT $\mathrm{BD}_{1}$, we synthesized BacPROTAC-3 linking sCym-1 and JQ1 (Figure 5A). The degrader was able to recruit BRDT ${ }_{\mathrm{BD} 1}$ (Figure S5D-F) and induce its degradation by ClpC1P1P2 in a highly specific manner (Figure 5B and S6). After validating the activity of the sCym-1 BacPROTAC-3 in vitro, 280 we investigated whether it was able to reprogram ClpC1P1P2 in a cellular environment. For this purpose, we used M. smegmatis cells stably expressing BRDT $_{\mathrm{BD} 1 .}$ We treated the culture with BacPROTAC-3 or alternatively, the individual building blocks sCym-1 and JQ1 (Figure 5C). After a 30-minute incubation, we quantified BRDT $T_{\mathrm{BD} 1}$ levels using capillary western blots. We found that BacPROTAC-

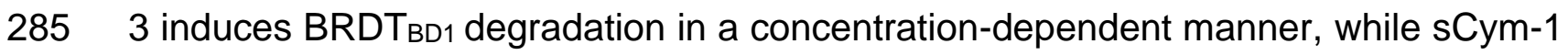
or JQ1 treatments did not significantly alter BRDT ${ }_{\mathrm{BD} 1}$ levels (Figure 5D and S7). Given the reported deregulation of ClpC1P1P2 by CymA (Maurer et al., 2019), we next assessed whether BacPROTAC-3 led to selective elimination of BRDTBD1 or had a global impact on the mycobacterial proteome. To address this point, we performed a tandem mass tag mass spectrometric (TMT-MS) analysis of $M$. smegmatis lysates. 
Isobaric labelling allowed the detection and quantification of 2912 proteins. Among

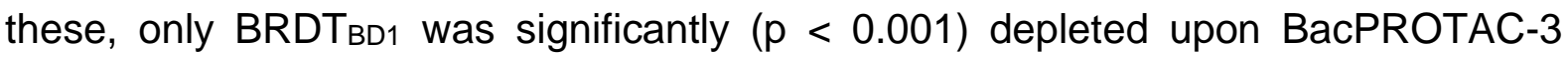
treatment (Figure 5E). Thus, the quantitative TMT-MS analysis provides compelling evidence that BacPROTAC-3 is capable of inducing degradation of the BRDTBD1

295 substrate in a highly specific manner. Together, our in vivo data demonstrate that CymA-based BacPROTACs can reprogram CIpC1P1P2 to induce POI degradation in mycobacteria. Furthermore, our data show that despite its chemical modification, the CymA scaffold maintains its ability to pass the mycobacterial cell envelope, providing an attractive tool for BacPROTAC development.

\section{DISCUSSION}

Since resistance of pathogenic bacteria to current treatments is outpacing discovery of new antibiotics, it is crucial to expand the number of effective antimicrobial drugs. In the present work, we pioneered a methodology to develop next generation antibiotics. We developed small-molecule degraders that extend the PROTAC 305 approach to bacteria. The resulting BacPROTACs reprogram the pArg-dependent ClpCP protease, the functional equivalent of the eukaryotic proteasome, and target it toward a specific substrate. As seen for pArg- and CymA-derived anchors, various molecules that bind to the substrate receptor of the ClpCP protease can be incorporated into BacPROTACs. By using a cell-permeable degrader, we also

310 demonstrate that recruitment of a model protein to the mycobacterial ClpC1P1P2 protease leads to selective protein degradation in the bacterial cell. This in vivo analysis emphasizes the feasibility of applying the BacPROTAC approach to pathogens. The ClpC-directed degraders can be applied to Gram positive bacteria and mycobacteria, and can thus target pathogens that are a serious global health threat,

315 including M. tuberculosis and Methicillin-resistant S. aureus (Lewis, 2020). Given the similar structural organization of the ClpAP protease, which also employs the Nterminal domain of the double ring ATPase CIpA as a substrate receptor, we presume that the BacPROTAC strategy presented here can be expanded to related Clp proteolytic complexes and used to combat the most severe pathogens across bacterial 320 phyla.

Analogous to PROTACs active in eukaryotic cells, the bacterial degraders should have certain benefits over standard antibiotics that inhibit a limited group of microbial 
enzymes. The modular scaffold of BacPROTACs, for example, could allow many different POls to be targeted for degradation. Currently, over 90,000 compounds directed against more than 70 different M. tuberculosis proteins are reported in the $\mathrm{CHEMBL}$ database. Connecting these POI ligands to $\mathrm{ClpC} 1$ binders would allow systematic evaluation of their potential to induce degradation of specific M. tuberculosis targets. The incorporated POI ligands do not need to occupy a functional site, and can thus target transcription factors, structural proteins and enzymes alike. High affinity binders that were identified in previous drug discovery efforts, but failed to block protein function, could therefore be repurposed as BacPROTAC building blocks and increase the number of druggable bacterial targets. Importantly, the developed approach could be employed to target virtually any bacterial protein since host-pathogen selectivity is achieved by reprogramming the

335 ClpCP protease, which is absent in human cells. Beside targeting essential bacterial proteins, the developed approach could be used to eliminate certain virulence proteins. Reducing the evolutionary pressure to develop antibiotic resistance would be particularly interesting in combinatorial treatments with established antimycobacterial drugs. It should be also noted that the bacterial degraders seem to have a mechanistic 340 advantage over their eukaryotic analogs. While PROTACs need to manipulate the complex E1-E2-E3 ubiquitination system to assemble a certain ubiquitin chain on a properly located lysine residue of the substrate, the bacterial degraders operate in a more straightforward manner. They influence the degradation reaction itself, bringing substrates directly to the ClpCP protease. We thus presume that optimizing degrader 345 efficiency and finding suitable substrates could be facilitated, streamlining the rational design of potent antibacterial compounds.

The possibility of eliminating a POI from the cell by addition of a chemical compound has broad applications for basic research as well. The function of proteins in prokaryotic and eukaryotic cells is often investigated by studying knock-out strains.

350 However, genetic manipulation is difficult when dealing with essential proteins, and requires special strategies that allow conditional, reversible and temporal control of protein levels. Chemical biology approaches that leverage the potency of small molecule degraders help to overcome these limitations (Burslem and Crews, 2020). Prominent examples are the auxin-inducible degron (AID) (Nishimura et al., 2009) and the selective removal of HaloTag fusion proteins (Neklesa et al., 2011). Likewise, the 
selective degradation of BRDTBD1-POI fusion proteins by BacPROTACs represents an attractive approach to generate conditional knockdowns in mycobacteria, allowing to dissect the function of essential proteins. These essential proteins may also be the very factors that are likely to become viable and effective targets for novel antimicrobials. In addition to addressing the biology of important microbial proteins, the degraders could thus help to validate antibiotic targets. As seen for small-molecule degraders in eukaryotic cells, we anticipate that BacPROTACs have the potential to accelerate drug discovery against pathogenic bacteria and facilitate the rational design of antibiotics targeting a wide range of essential microbial targets that were previously considered undruggable. 


\section{Acknowledgements}

We thank all members of the Clausen group for remarks on the manuscript and our colleagues Jan-Michael Peters and Yasin Dagdas for providing feedback on this study. Editing support was provided by Life Science Editors. This work was supported by the European Research Council (AdG 694978, T.C.), an FFG Headquarter Grant (No 852936, T.C.), the Vienna Science and Technology Fund (WWTF LS17-029, F.E.M.) and Boehringer Ingelheim (Research Beyond Border program, J.L.). F.E.M. and J.L.

375 are members of the Boehringer Ingelheim Discovery Research global post-doc program. The IMP is supported by Boehringer Ingelheim.

\section{Author contributions}

F.E.M., A.M., M.K. and T.C. designed experiments; S.K. performed the chemical synthesis of the BacPROTACs, F.E.M. performed biochemical assays and binding measurements; F.E.M and D.H. performed the cryo-EM analysis, A.M. and J.K the crystallographic analysis; J.L., S.O. and R.K. prepared bacterial strains and carried out the in vivo analysis; D.M.H. and M.H. performed the mass spectrometry analysis; T.C. coordinated the research project and prepared the manuscript together with 385 F.E.M.

\section{Declaration of interests}

F.E.M., S.K., J.L., A.M., M.K. and T.C. are named as inventors of a currently filed patent that is based on the presented findings. 


\section{FIGURES}
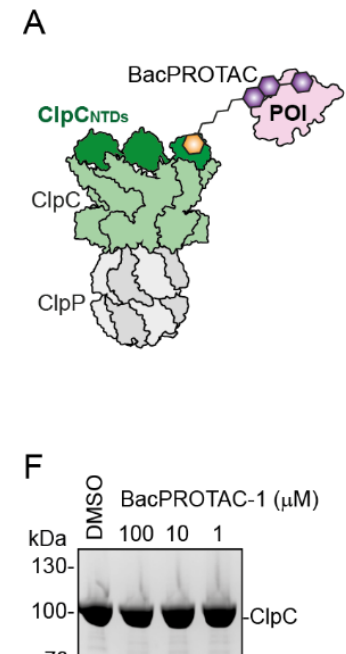

395

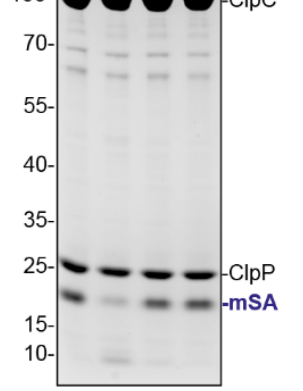

B

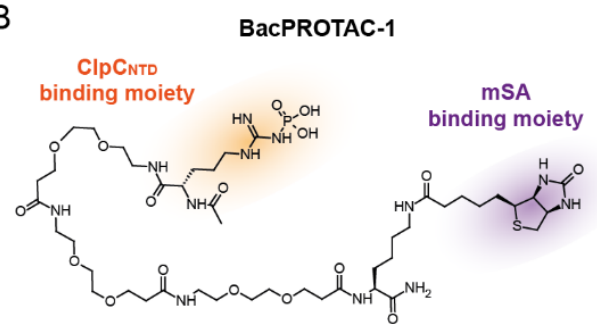

C
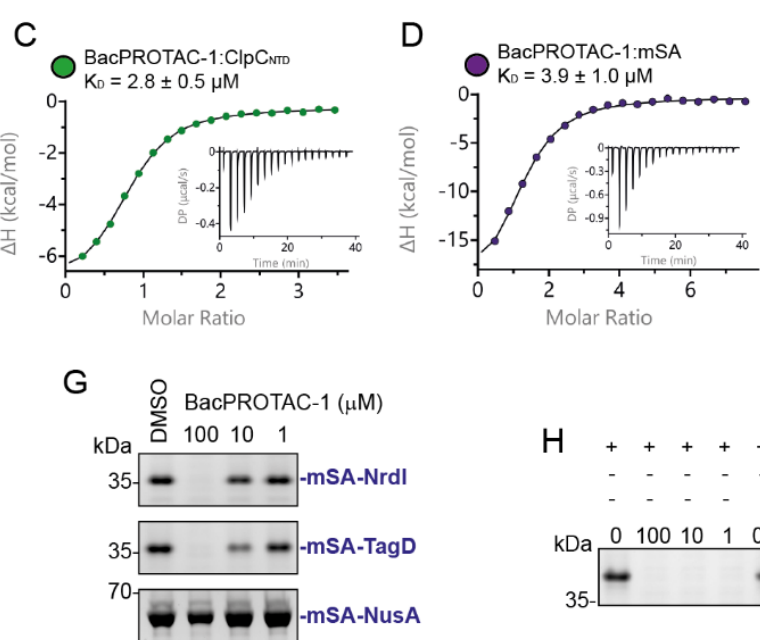

$\mathrm{E}$
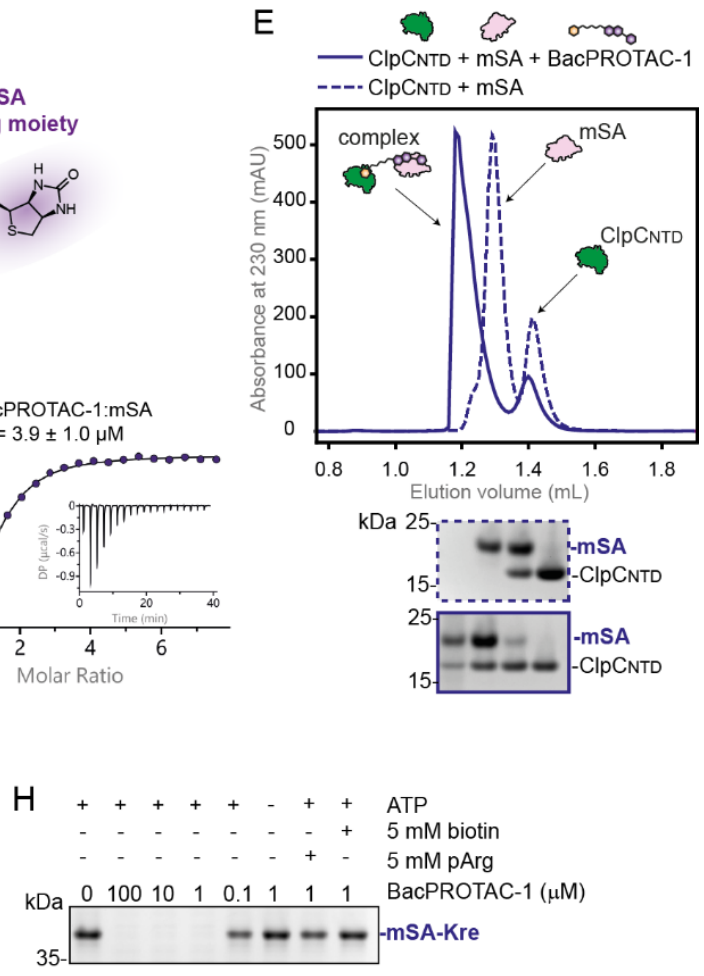

Figure 1. In vitro reprogramming of $B$. subtilis ClpCP by BacPROTAC-1.

(A) Schematic representation of the BacPROTAC approach, hijacking the ClpCP protease. (B) Chemical structure of BacPROTAC-1, designed to tether the model

400 substrate $\mathrm{mSA}$ to the $\mathrm{ClpC}_{\mathrm{NTD}}$ receptor domain. (C, D) ITC titrations of BacPROTAC1 against ClpCNTD and MSA. Calculated KD's and their standard deviation are indicated $(n \geq 3)$. (E) SEC analysis of a stoichiometric mSA:ClpC $C_{N T D}$ mixture in the presence (solid line) or absence (dashed line) of BacPROTAC-1. An SDS-PAGE analysis (lower panel) confirms the formation of the indicated ternary complex. (F-H) SDS-PAGE 405 analysis of in vitro degradation assays. $\mathrm{mSA}$ alone and $\mathrm{mSA}$ fusion proteins (mSANrdl, mSA-TagD, mSA-NusA, mSA-Kre) are degraded by CIpCP in a BacPROTAC-1dependent manner (2 hours incubation, DMSO used as control). (H) For mSA-Kre, further controls indicate that degradation is ATP-dependent and that pArg or biotin block the BacPROTAC-1-induced degradation. Uncropped images of SDS-PAGE gels are shown in Figure $\mathbf{S 8 .}$ 

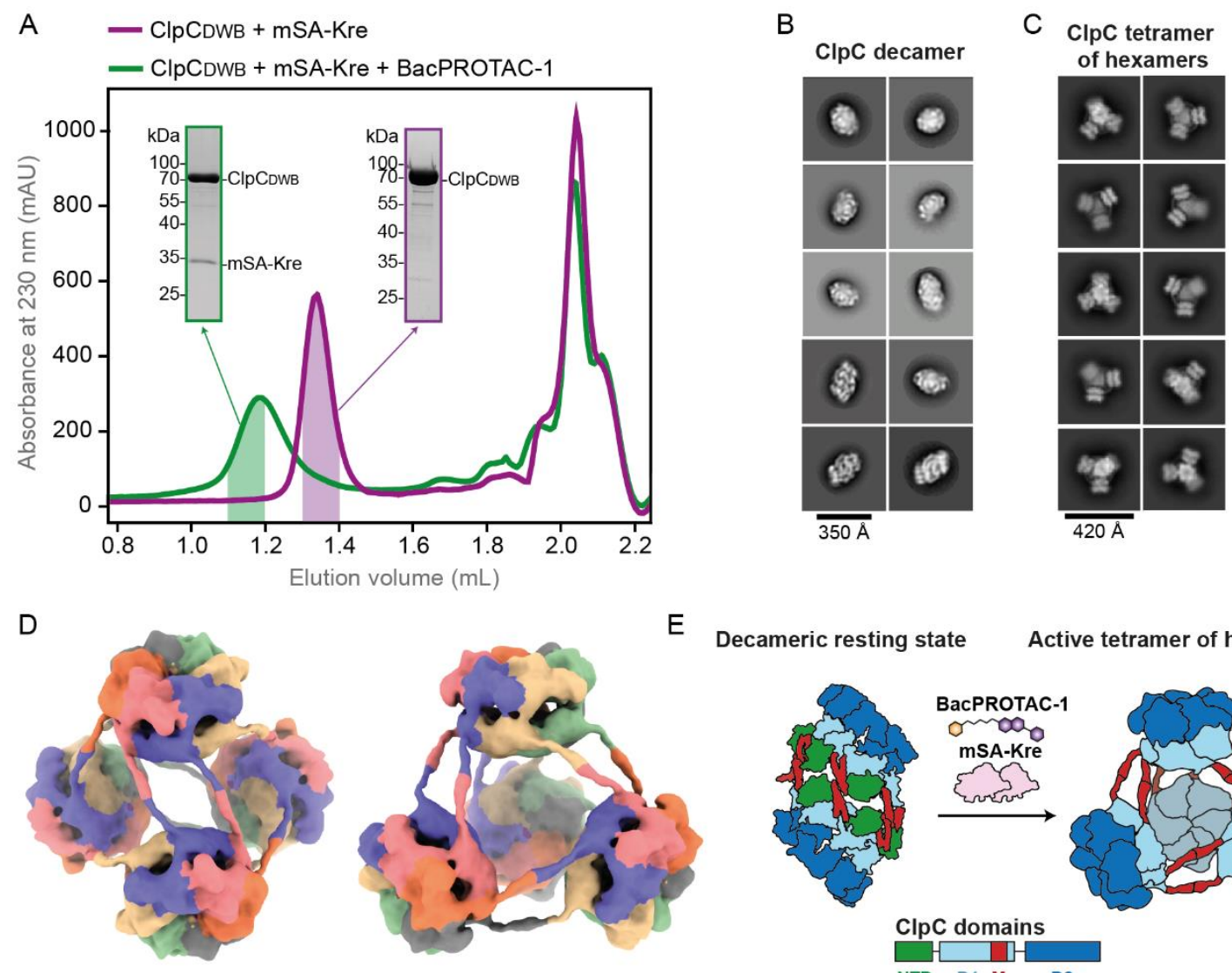

Decameric resting state

Active tetramer of hexamers

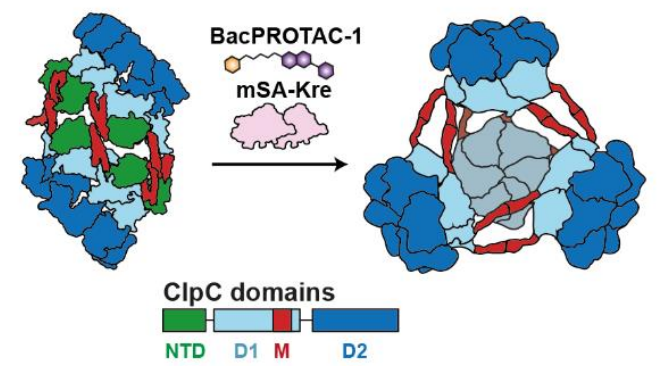

Figure 2. BacPROTAC-1 induces formation of an active ClpC oligomer.

(A) SEC analysis of a stoichiometric ClpCowB:mSA-Kre mixture in the presence (green) or absence (magenta) of BacPROTAC-1. The fractions used for EM analysis

415 are highlighted. SDS-PAGE gels illustrate the coelution of ClpCDwB and mSA-Kre in the presence of BacPROTAC-1. (B) Representative 2D class averages obtained from negative stained EM images, showing that $B$. subtilis $\mathrm{ClpC}$ forms a decameric complex representing the resting state. (C) Representative $2 \mathrm{D}$ class averages showing that in the presence of substrate and BacPROTAC-1, ClpC transforms into a 24-mer, composed of four hexamers present in functional form. (D) Refined 3D model (10 resolution) of the tetramer of $\mathrm{ClpC}$ hexamers, shown in two orientations. Individual ClpC protomers are colored differently. (E) Schematic representation of the BacPROTAC-induced conversion of the inactive ClpC decamer into the active higherorder particle (24-mer), using a domain-based coloring mode. 


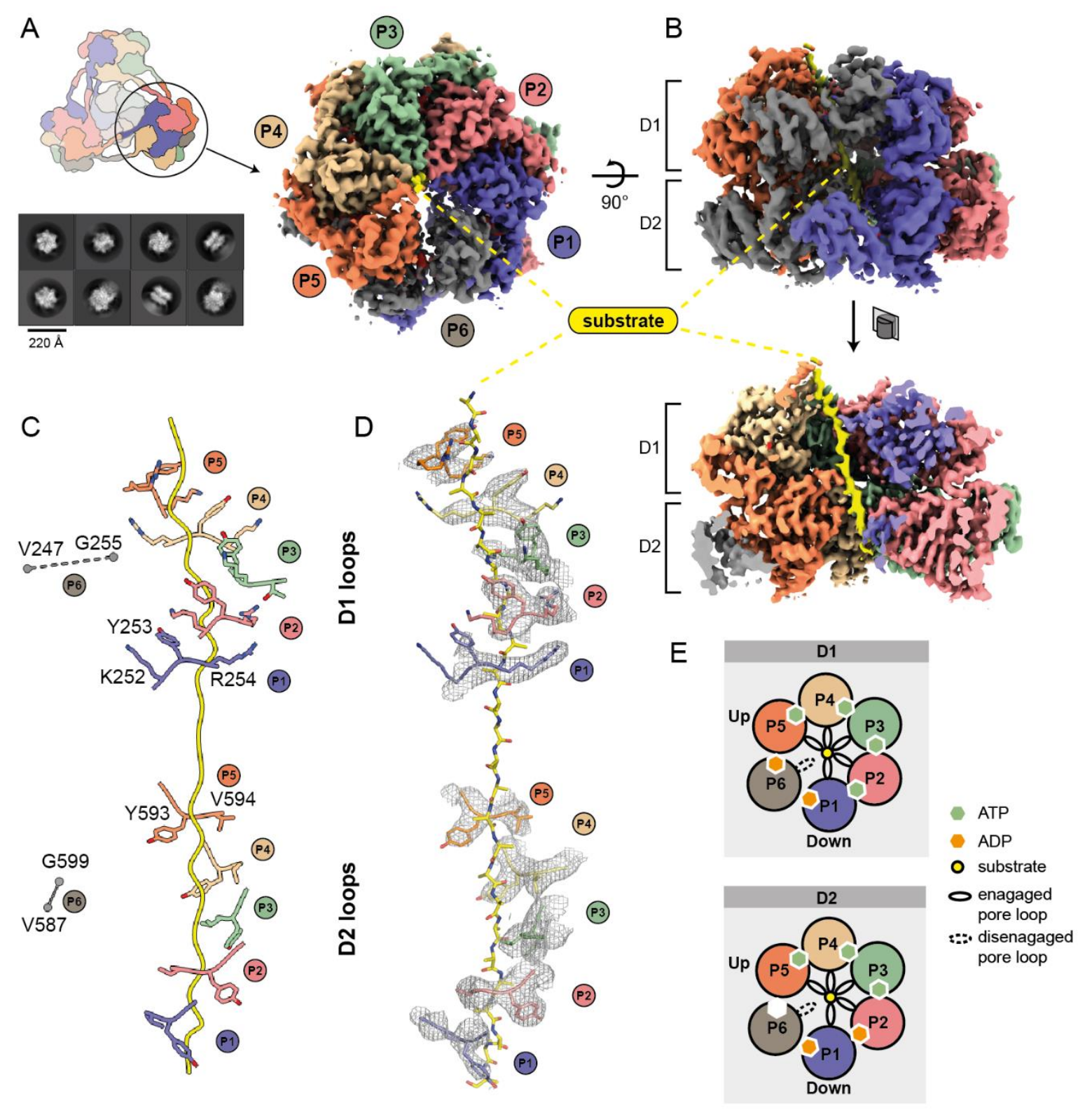

Figure 3. Cryo-EM structure of the activated ClpC hexamer in complex with a

\section{BacPROTAC-tethered substrate.}

(A) Representative 2D class averages are shown together with the final cryo-EM map,

430 having a resolution of $3.7 \AA$. The density is colored according to subunits that are termed $\mathrm{P} 1$ to $\mathrm{P} 6$. The substrate captured in the central channel is shown in yellow. (B) Side view of the substrate-bound ClpC. The lower panel shows the cross section of the hexamer highlighting the substrate threaded through the two D1 and D2 rings of ClpC. The substrate was well defined by cryo-EM density over the entire passage 435 of the central channel ( $80 \AA$ A). (C) Arrangement of primary D1 and D2 pore loops engaging the substrate (peptide backbone shown in yellow). The P6 pore loops, which 
440 representation of nucleotide states and substrate engagement of the six ClpC protomers. Nucleotides were assigned based on cryo-EM density and distance matrices in the active site (Figure S3). 


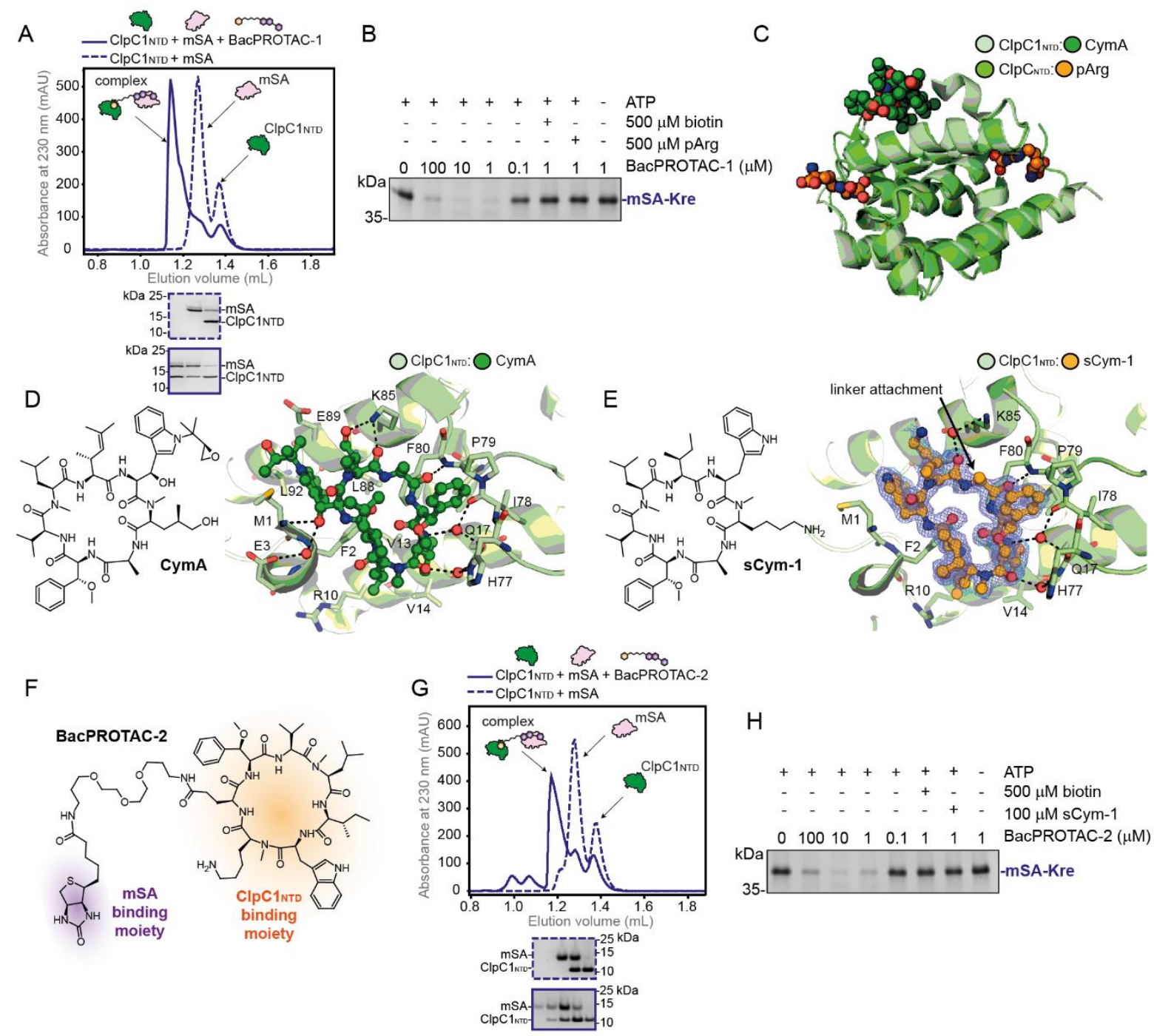

Figure 4. BacPROTACs can reprogram the mycobacterial CIpC1P1P2.

(A) SEC analysis of a stoichiometric mSA:CIpC1NTD mixture in the presence (solid line) or absence (dashed line) of BacPROTAC-1. SDS-PAGE analysis (lower panel) confirms the formation of the indicated ternary complex. (B) SDS-PAGE analysis of mSA-Kre degradation in vitro, following a 2-hour incubation with $M$. smegmatis ClpC1P1P2. The pArg-containing BacPROTAC-1 induces degradation in a concentration-dependent manner and can be outcompeted by separately provided pArg or biotin. (C) Superposition of CIpC NTD:pArg (PDB: 5HBN) with CIpC1 $1_{\text {NTD:CymA }}$ 455 (PDB: 3WDC) crystal structures highlights the distinct locations of the ligand binding sites. (D) Chemical structure of CymA and co-crystal structure with ClpC1NTD (PDB: 3WDC). Dotted lines represent hydrogen bonds, water molecules are shown as red spheres. (E) Chemical structure of the CymA analogue sCym-1 and co-crystal 
structure with CIpC1NTD (PDB: 7AA4, this study), overlaid with the $\mathrm{F}_{0}-\mathrm{F}_{\mathrm{c}}$ electron density map of the ligand (calculated at $1.7 \AA$ resolution, contoured at $2 \sigma$ ). The arrow indicates the position for attaching the BacPROTAC linker. The respective alanine side chain does not contribute to the sCym-1:ClpC1NTD interface. (F) Chemical structure of BacPROTAC-2, employing sCym-1 as a distinct ClpC1 anchor point. (G) SEC analysis of a stoichiometric mSA:CIpC1NTD mixture in the presence (solid line) or absence 465 (dashed line) of BacPROTAC-2. An SDS-PAGE analysis (lower panel) confirms the formation of the indicated ternary complex. $(\mathbf{H})$ In vitro degradation of $\mathrm{mSA}-\mathrm{Kre}$ in the presence of BacPROTAC-2, using the same assay conditions as in $(A)$. 
A

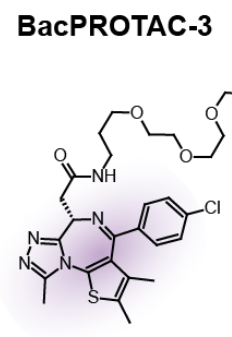

BRDTвD1 binding moiety

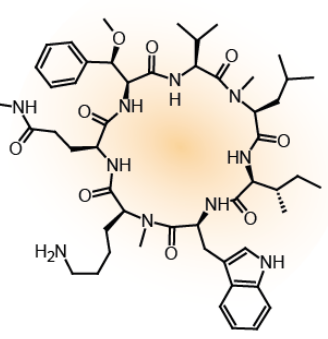

CIpC1NTD binding moiety

B

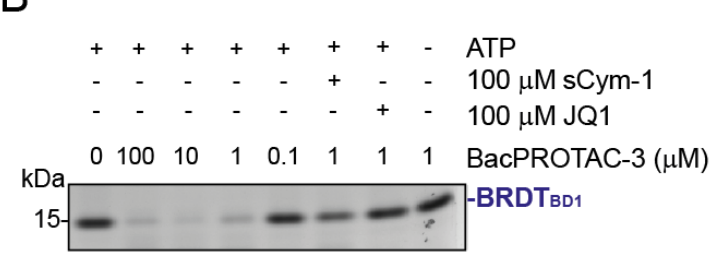

D

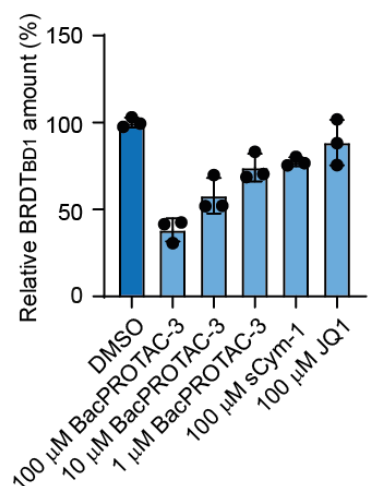

$E$

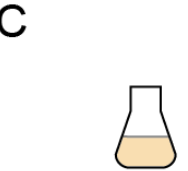
M. smegmatis

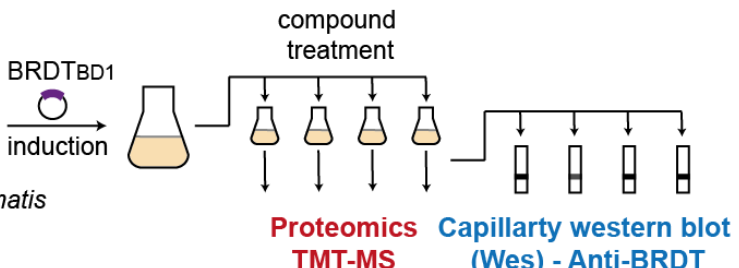
TMT-MS (Wes) - Anti-BRDT
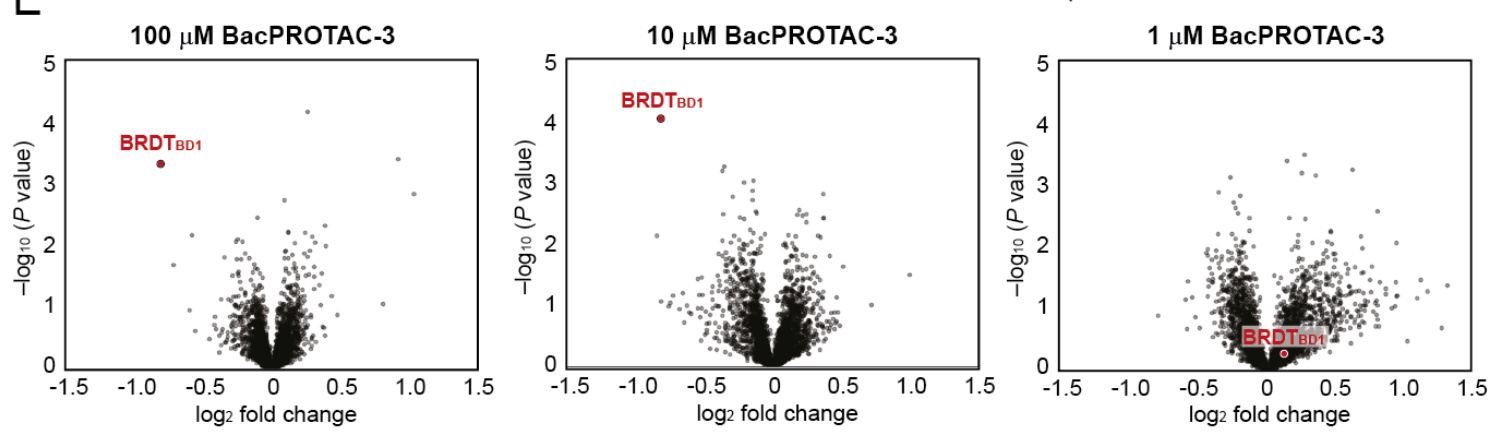

Figure 5. BacPROTAC-3 mediates degradation of a POI in mycobacteria.

(A) Chemical structure of BacPROTAC-3 bridging JQ1 and SCym-1. (B) SDS-PAGE analysis of in vitro degradation following a 2-hour incubation with $M$. smegmatis CIpC1P1P2. BacPROTAC-3 promotes degradation of BRDT $\mathrm{BD}_{1}$ in a concentration-

475 dependent manner and can be blocked by addition of its individual head groups. (C) Outline of BRDT $\mathrm{BD}_{\mathrm{B} 1}$ degradation assay in mycobacteria. (D) Capillary Western blot (Wes) analysis of BacPROTAC-3 mediated effects on BRDT $\mathrm{BD} 1$ in the cell after 30 minutes of incubation. The bar chart summarizes three independent experiments,

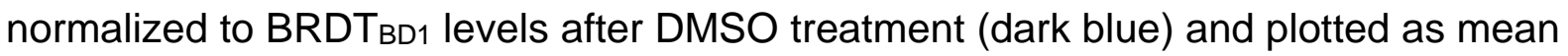
$480 \pm$ SD. (E) TMT-MS proteomics analysis of the BacPROTAC-3 effect after 2 hours of incubation. The volcano plots show the fold-change $\left(\log _{2}\right)$ in protein abundance in comparison to DMSO treatment, plotted against $P$ value $\left(-\log _{10}\right)$ (two-tailed Student's T-test; triplicate analysis). The BRDT ${ }_{\mathrm{BD} 1}$ protein is highlighted in red. 
Table 1. Cryo-EM data collection, refinement and validation statistics

\begin{tabular}{|c|c|c|}
\hline & $\begin{array}{l}\text { ClpC hexamer } \\
\text { (EMDB-11707) } \\
\text { (PDB 7ABR) }\end{array}$ & $\begin{array}{l}\text { Tetramer of CpC } \\
\text { hexamers } \\
\text { (EMDB-11708) }\end{array}$ \\
\hline \multicolumn{3}{|l|}{ Data collection and processing } \\
\hline Magnification & 75,000 & 75,000 \\
\hline Voltage (kV) & 300 & 300 \\
\hline Electron exposure $\left(\mathrm{e}-/ \AA^{2}\right)$ & 54 & 54 \\
\hline Defocus range $(\mu \mathrm{m})$ & $1.3-4.5$ & $1.3-4.5$ \\
\hline Pixel size $(\AA ̊)$ & 1.058 & 1.058 \\
\hline Symmetry imposed & C1 & $T$ \\
\hline Initial particle images (no.) & $1,034,627$ & $1,173,558$ \\
\hline Final particle images (no.) & 212,314 & 87,575 \\
\hline Map resolution $(\AA ̊)$ & 3.7 & 10 \\
\hline FSC threshold & 0.143 & 0.143 \\
\hline Map resolution range $(\AA)$ & $3.4-5.5$ & \\
\hline \multicolumn{3}{|l|}{ Refinement } \\
\hline Initial model used (PDB code) & $3 J 3 U$ & \\
\hline Model resolution $(\AA)$ & 3.7 & \\
\hline FSC threshold & 0.5 & \\
\hline \multicolumn{3}{|l|}{ Model resolution range $(\AA)$} \\
\hline Map sharpening $B$ factor $\left(\AA^{2}\right)$ & -210 & \\
\hline \multicolumn{3}{|l|}{ Model composition } \\
\hline Non-hydrogen atoms & 27,209 & \\
\hline Protein residues & 3445 & \\
\hline Ligands & ADP: 4 , ATP: 7 & \\
\hline \multicolumn{3}{|l|}{$B$ factors $\left(\AA^{2}\right)$} \\
\hline Protein & 67.93 & \\
\hline
\end{tabular}


bioRxiv preprint doi: https://doi.org/10.1101/2021.06.09.447781; this version posted June 10, 2021. The copyright holder for this preprint (which was not certified by peer review) is the author/funder, who has granted bioRxiv a license to display the preprint in perpetuity. It is made available under aCC-BY-NC-ND 4.0 International license.

\begin{tabular}{|c|l|l|}
\hline Ligand & 53.21 & \\
\hline $\begin{array}{l}\text { R.m.s. deviations } \\
\text { Bond lengths }(\AA)\end{array}$ & 0.011 & \\
\hline Validation & 1.221 & \\
MolProbity score $\left(^{\circ}\right)$ & & \\
Clashscore & 2.33 & \\
Poor rotamers (\%) & 19.31 & \\
\hline Ramachandran plot & 0.51 & \\
Favored (\%) & & \\
Allowed (\%) & 90.13 & \\
Disallowed (\%) & 9.64 & \\
\hline EMRinger score & 0.24 \\
\hline
\end{tabular}




\section{MATERIALS AND METHODS}

\section{DNA constructs.}

Cloning of constructs for $E$. colioverexpression of full-length $B$. subtilis ClpC, ClpCDWB (E280A/E618A), CIpCNTD (1-148), ClpP, and BRDTBD1 was previously described (Filippakopoulos et al., 2012; Trentini et al., 2016). In case of expression constructs of B. subtilis ClpC, ClpC $C_{D W B}, C_{N T D}$ and $\mathrm{ClpP}$, all variants were fusion constructs 495 containing a C-terminal hexahistidine tag, except that for BRDT ${ }_{\mathrm{BD} 1}$ which contained a $\mathrm{N}$-terminal hexahistidine tag followed by a TEV cleavage site.

Synthetic DNA of M. smegmatis clpP1, clpP2 and clpC1 genes (MSMEG_4673, MSMEG_4672, MSMEG_6091) was ordered from GeneArt (Thermo Fisher) and cloned into a pET21a vector DNA. CIpP1 and CIpP2 expression constructs encode a

500 C-terminal tetrahistidine tag fusion, while that of $\mathrm{ClpC} 1$ expresses wild type protein. The coding region of $M$. smegmatis clpC1 NTD (1-148) was cloned into pET21a and encodes a C-terminal hexahistidine tag fusion.

DNA for mSA-fusion constructs of three B. subtilis proteins (mSA-Nrdl, mSA-TagD, mSA-NusA) cloned into a pETM14 vector was purchased from Genewiz. Expression

505 constructs of $\mathrm{mSA}$ and $\mathrm{mSA}-\mathrm{Kre}$ were cloned into a pNIC28-Bsa4 vector. These constructs encode a $\mathrm{N}$-terminal hexahistidine tag followed by a TEV cleavage site fusion and a glycine-serine linker introduced between $\mathrm{mSA}$ and the bacterial protein. The mSA-Kre construct used for cryo-EM analysis was cloned into pET21a vector DNA and encodes a C-terminal hexahistidine tag fusion (Table S2 reports the

510 translated amino acid sequences of all mSA-fusion proteins).

Plasmid DNA for BRDT $\mathrm{BD}_{1}$ expression in $M$. smegmatis was ordered from Genescript. It contains the coding region of BRDT $\mathrm{BD}_{1}$ cloned into $\mathrm{pMyC}$ vector DNA. pMyC was a gift from Annabel Parret \& Matthias Wilmanns (Addgene plasmid \# 42192; http://n2t.net/addgene:42192 ; RRID:Addgene_42192).

\section{Protein expression and purification.}

Plasmid DNA was transformed into E. coli BL21 (DE3) or Rosetta cells (for $M$. smegmatis ClpC1, ClpP1 and ClpP2 proteins) and grown in LB broth supplemented with the respective antibiotic at $37^{\circ} \mathrm{C}$. Protein expression was induced by adding 0.1 - 
$0.5 \mathrm{mM}$ isopropyl-1-thio- $\beta-\mathrm{D}-$ galactopyranoside (IPTG) at an $\mathrm{OD}_{600}$ of 0.8 . Cells were cultured overnight at $18-20{ }^{\circ} \mathrm{C}$, harvested by centrifugation and either lysed by sonication in a buffer containing $500 \mathrm{mM} \mathrm{NaCl}, 50 \mathrm{mM}$ Tris pH 7.5, $10 \mathrm{mM}$ imidazole, and $0.25 \mathrm{mM}$ tris(carboxyethyl)phosphine (TCEP), or flash frozen and stored at $-80^{\circ} \mathrm{C}$ until purification.

Cell debris was removed by centrifugation. His-tagged proteins were purified from the 525 cleared supernatants using Ni-NTA Agarose beads in batch. After several washing steps, bound protein was eluted using $50 \mathrm{mM}$ Tris $\mathrm{pH}$ 7.5, $100 \mathrm{mM} \mathrm{NaCl}, 300 \mathrm{mM}$ imidazole, $0.25 \mathrm{mM}$ TCEP. Eluted protein was loaded onto a size exclusion chromatography column (Superdex 75 16/60 or Superdex 200 16/60 (GE Healthcare) depending on protein size) equilibrated in $50 \mathrm{mM}$ Tris pH 7.5, $100 \mathrm{mM} \mathrm{NaCl}$. For ClpC

530 and ClpCDWB purifications, the salt concentration of the size exclusion buffer was increased to $300 \mathrm{mM} \mathrm{NaCl}$. Purified fractions were pooled and concentrated before flash freezing and stored at $-80^{\circ} \mathrm{C}$.

Cell pellets for ClpP1 and ClpP2 purification were resuspended in 50 mM HEPES$\mathrm{NaOH} \mathrm{pH}$ 7.8, $300 \mathrm{mM} \mathrm{NaCl}, 30 \mathrm{mM}$ imidazole and lysed by sonication. Cleared 535 lysates were purified by Ni-NTA affinity chromatography (elution buffer: $50 \mathrm{mM}$ HEPES-NaOH, pH 7.8, $300 \mathrm{mM} \mathrm{NaCl}, 250 \mathrm{mM}$ imidazole) and subsequently by gel filtration using a Superose $616 / 60$ column (GE Healthcare) equilibrated with $50 \mathrm{mM}$ HEPES-NaOH pH 7.2, $150 \mathrm{mM} \mathrm{KCl.} \mathrm{10 \%} \mathrm{(} / v / v)$ glycerol was added to the elution fractions before flash freezing and storage at $-80^{\circ} \mathrm{C}$. Processing of the full-length

540 ClpP1 and ClpP2 to the mature ClpP1P2 complex was performed similar as previously described by Leodolter et al. (Leodolter et al., 2015).

Cell pellets for $\mathrm{ClpC} 1$ purification were resuspended in $50 \mathrm{mM}$ Tris $\mathrm{pH} 7.5,75 \mathrm{mM} \mathrm{KCl}$, 2 mM EDTA, 10\% ( $/ v)$ glycerol and lysed by sonication. After clarification of the lysate, ClpC1 was precipitated using 40\% ( $w / v)$ ammonium sulphate. The pellet was 545 resuspended in lysis buffer, loaded on a HiLoad 26/10 Q Sepharose High Performance column (GE Healthcare) equilibrated with lysis buffer and eluted in a gradient to $1 \mathrm{M}$ $\mathrm{KCl}$. ClpC1-containing fractions were pooled and precipitated again using $40 \%(\mathrm{~W} / \mathrm{V})$ ammonium sulphate. The pellet was resuspended in $50 \mathrm{mM}$ HEPES-NaOH pH 7.2, $150 \mathrm{mM} \mathrm{KCl}, 10 \%(\mathrm{~V} / \mathrm{v})$ glycerol and loaded on a HiLoad 16/10 Superdex 200 prep 550 grade gel filtration column (GE Healthcare) equilibrated in the same buffer. ClpC1 containing fractions were pooled and stored at $-80^{\circ} \mathrm{C}$. Protein purity was monitored 
by Coomassie stained SDS-PAGE and correct molecular mass of purified proteins was verified by mass spectrometry.

\section{Isothermal Titration Calorimetry (ITC).}

555 ITC experiments were performed using a MicroCal PEAQ-ITC instrument (Malvern) at $25^{\circ} \mathrm{C}$ in a buffer containing $50 \mathrm{mM}$ Tris $\mathrm{pH} 7.5,100 \mathrm{mM} \mathrm{NaCl}$. Each titration consisted of 19 injections with intervals of $120 \mathrm{~s}$ (the first injection of $0.4 \mu \mathrm{L}$ was followed by 18 injections of $2 \mu \mathrm{L}$ ) at constant stirring at $750 \mathrm{rpm}$. DMSO concentration was matched between cell and syringe to be $2 \%(v / v)$. Data were fitted using a single binding site

560 model with a fitted offset subtraction using the MicroCal PEAQ-ITC Analysis Software. Each titration was repeated at least twice.

Titrations involving BacPROTAC-1 were performed with the ligand loaded into the syringe and the protein into the cell. $400 \mu \mathrm{M}$ BacPROTAC-1 was titrated against 20 $\mu \mathrm{M} \mathrm{CIPC}_{\mathrm{NTD}}$ or mSA. A control titration of ligand into buffer was performed in order to

565 determine the heat of dilution.

Titrations involving sCym-1, BacPROTAC-2 and BacPROTAC-3 were performed with the ligand loaded into the cell and protein into the syringe to account for the low solubility of the compounds in aqueous solutions.

\section{Analytical size exclusion chromatography (SEC)}

570 For the analytical runs involving $\mathrm{CIpC/C1NTD}$ and $\mathrm{mSA}$ or BRDT ${ }_{\mathrm{BD} 1}$, the proteins were premixed at equimolar concentrations $(25 \mu \mathrm{M})$ and BacPROTAC-1, BacPROTAC-2, BacPROTAC-3 $(25 \mu \mathrm{M})$ dissolved in 100\% ( $/ v / v)$ DMSO giving rise to a final concentration of $0.25 \%(v / v)$. For control experiments only DMSO was added to that concentration. Samples were loaded into a $30 \mu \mathrm{L}$ loop and applied to a Superdex 75

$5753.2 / 300$ increase column (GE healthcare) equilibrated in $50 \mathrm{mM}$ Tris pH 7.5, $100 \mathrm{mM}$ $\mathrm{NaCl}$. Runs were performed at room temperature at a flow rate of $0.06 \mathrm{~mL} / \mathrm{min}$. Each run was performed in triplicate. $100 \mu \mathrm{L}$ fractions were collected and analyzed by SDSPAGE and Coomassie staining.

For electron microscopy analysis, B. subtilis ClpCDwB $(25 \mu \mathrm{M}$ monomer) and the 580 substrate mSA-Kre $(25 \mu \mathrm{M})$ were premixed in a buffer containing $50 \mathrm{mM}$ Tris $\mathrm{pH} 7.5$, $50 \mathrm{mM} \mathrm{KCl}, 5 \mathrm{mM} \mathrm{MgCl}, 5 \mathrm{mM}$ ATP and $1.25 \%$ ( $/ \mathrm{v})$ DMSO or $156 \mu \mathrm{M}$ BacPROTAC- 
585 temperature at a flow rate of $0.06 \mathrm{~mL} / \mathrm{min}$. $100 \mu \mathrm{L}$ fractions were collected and used to prepare grids for EM analysis (Figure 2A).

\section{In vitro degradation assays}

In vitro degradation assays containing $0.5 \mu \mathrm{M} B$. subtilis ClpC (hexameric), $0.5 \mu \mathrm{M} B$. subtilis ClpP (heptameric), $2 \mu \mathrm{M}$ substrate protein, $15 \mathrm{mM}$ phosphoenolpyruvate

590 (PEP), $10 \mathrm{U} / \mathrm{mL}$ pyruvate kinase (Sigma Aldrich) were performed in a buffer containing $50 \mathrm{mM}$ Tris pH 7.5 (at $37^{\circ} \mathrm{C}$ ), $50 \mathrm{mM} \mathrm{KCl,} 20 \mathrm{mM} \mathrm{MgCl}, 10 \%$ ( $/ \mathrm{v}$ ) glycerol. Compounds were dissolved in 100\% ( $/ v)$ DMSO and further diluted giving rise to a final concentration of $1 \%(v / v)$ of DMSO in the assay. In control experiments DMSO was added to that concentration. Reactions were started by addition of 5 mM ATP and

595 terminated by adding SDS sample buffer after 2 hours incubation at $37^{\circ} \mathrm{C}$. The samples were analyzed by SDS-PAGE and Coomassie staining. Degradation assays using $0.5 \mu \mathrm{M}$ M. smegmatis ClpC1 (hexameric), $0.25 \mu \mathrm{M} M$. smegmatis mature ClpP1P2 (both heptameric), $2 \mu \mathrm{M}$ substrate protein, $15 \mathrm{mM}$ PEP, $10 \mathrm{U} / \mathrm{mL}$ pyruvate kinase (Sigma Aldrich) were carried out in 50 mM HEPES-NaOH pH 7.2, $100 \mathrm{mM} \mathrm{KCl}$, $60010 \mathrm{mM} \mathrm{MgCl}_{2}, 10 \%(\mathrm{v} / \mathrm{v})$ glycerol using the same procedure as described above. All experiments were performed in triplicates which gave similar results.

\section{Negative staining EM sample preparation, data collection and processing.}

In the absence of BacPROTAC-1, CIpCDwB and the substrate mSA-Kre eluted

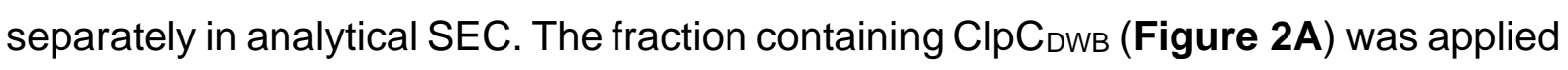
605 onto glow-discharged carbon-coated Cu/Pd Hexagonal 400 mesh grids (Agar Scientific) subsequently stained with a solution of $2 \%(w / v)$ uranyl acetate. The grids were screened and then imaged using a FEI Tecnai G2 20 microscope equipped with a 4k Eagle camera (FEI) using a pixel size of $1.8 \AA$ $/$ px. 1,004 micrographs were collected (a representative micrograph is shown in Figure S2A) and 856 particles

610 were manually picked and 2D classified, generating templates for subsequent automatic particle picking on the entire set of micrographs. 369,415 picked particles 
were extracted using box size of $300 \mathrm{px}$ and subjected to $2 \mathrm{D}$ classification. The resulting highest populated $2 \mathrm{D}$ class averages are shown in Figure 2B.

\section{Cryo-EM sample preparation and data collection.}

615 The ClpCDwB:BacPROTAC-1:mSA-Kre complex was isolated using analytical SEC. Cryo-EM grids were prepared using glow discharged R2/2 Cu 200 mesh grids (Quantifoil) pre-floated onto custom-made $2.9 \mathrm{~nm}$ continuous carbon film. $4 \mu \mathrm{L}$ sample were applied onto a grid held in the sample grid chamber of a Leica EM GP instrument (Leica Microsystem Inc.) cooled at $4{ }^{\circ} \mathrm{C}$, with a relative humidity of $80-90 \%$. Grids were

620 blotted $2 \mathrm{~s}$ with a Whatman type 1 filter paper using the blotting sensor and flash frozen in liquid ethane. The quality of the grids was screened using a Thermo Scientific Glacios Cryo Transmission Electron Microscope equipped with a Falcon3 Direct Electron Detector. For the best grid, a dataset of 4,455 micrographs was collected (a representative micrograph is shown in Figure S2B) on a Titan Krios equipped with a

625 Falcon 3EC detector with a nominal magnification of 75,000 resulting in pixel size of $1.058 \AA / p x$. A total dose of $54 \mathrm{e}^{-/ \AA^{2}}$ was fractionated into 40 frames. 452 poor quality images (heavy contamination, poor ice quality or bad information transfer) were discarded after visual inspection, leaving 4,003 images for further analysis.

\section{Cryo-EM analysis of the ternary complex between CIPC, BacPROTAC-1 and}

\section{0 mSA: the tetramer of hexamers}

Multi-frame micrographs were analyzed in cryoSPARC v2 (Punjani et al., 2017). The full micrographs were motion corrected and their contrast transfer function (CTF) parameters were estimated. 482 particles were manually picked and 2D classified, generating a template for subsequent automatic particle picking on the entire set of

635 micrographs. 1,173,558 picked particles were extracted using box size of $500 \mathrm{px}$ and subjected to two rounds of $2 \mathrm{D}$ classification, selecting only the classes containing the full tetramer of hexamers for further analysis (Figure 2C). 87,575 "clean" particles were used for $a b$ initio model generation and subsequent homogeneous refinement applying T symmetry. The approximate resolution of the obtained map was $10 \AA$ as judged by Gold Standard Fourier shell correlation (FSC) (Figure S2C). 


\section{Cryo-EM analysis of the ternary complex between CIPC, BacPROTAC-1 and} mSA: the hexameric building block

For the analysis of the single hexamers, the same multi-frame micrographs were motion corrected and dose-weighted using MotionCor2 1.0.5 (Zheng et al., 2017).

645 Aligned micrographs were used for CTF estimation in Gctf 1.06 (Zhang, 2016). A subset of 10 micrographs was used for manual particle picking in crYOLO v1.3.5 (Wagner et al., 2019), the picking centers were the single hexamers rather than the tetramer of hexamers. Manually picked particles were used to train a model for automatic particle picking on the full micrographs set (160 px box size). A total of

650 1,034,627 auto-picked particles were imported in RELION 3.0 (Zivanov et al., 2018). Particles were extracted with $2 \times 2$ binning (box size 300 px, rescaled to $150 \mathrm{px}$ ) and subjected to two rounds of $2 \mathrm{D}$ classification. The particles belonging to the best $2 \mathrm{D}$ classes (Figure 3A) were re-extracted un-binned (box size 300 px) and 3D classified using an initial 3D model generated with cryoSPARC v2 (Punjani et al., 2017). After

$6553 D$ classification, 212,314 particles were selected for 3D refinement. The resolution was estimated with Gold Standard FSC, and B-factor sharpening was applied, both using the RELION postprocessing. The final map used for model building had an overall resolution of $3.7 \AA$. Local resolution was estimated in RELION and is shown in Figure S2E.

\section{Model building}

An initial model was built in Coot (Emsley et al., 2010) by rigid body fitting of secondary structure elements of one of the previously reported ClpC structures into the EM-map (PDB: 3J3U) (Liu et al., 2013). This model, however, required partial rebuilding and several steps of manual real-space refinement in Coot. Substrate-bound ClpB cryo-

665 EM structures (Deville et al., 2019) were used as reference, as mSA-Kre binding caused substantial conformational rearrangements in the unfoldase hexamer when compared to the apo form of ClpC reported previously (Liu et al., 2013; Wang et al., 2011). The substrate polypeptide chain was modelled as a poly-alanine. Phenix (Afonine et al., 2018) was used for further real-space refinement. The quality of the 670 model and model to map fitting were assessed using Phenix (Afonine et al., 2018), MolProbity (Williams et al., 2018) and EMRinger (Barad et al., 2015). Structural 
illustrations were prepared using either UCSF Chimera (Goddard et al., 2007), UCSF ChimeraX (Goddard et al., 2018), or Pymol (Schrodinger, 2015).

\section{ClpC1 ${ }_{\mathrm{NTD}}: \mathrm{sCym-1}$ co-crystallization and structure determination}

675 ClpC1NTD in a buffer containing 10 mM Tris-Cl pH 7.5, $100 \mathrm{mM} \mathrm{NaCl,} 1$ mM sCym-1, and $1 \%(v / v)$ DMSO was crystallized in a hanging drop, vapor diffusion setup at $15 \mathrm{mg} / \mathrm{mL}$ concentration using a reservoir solution containing $100 \mathrm{mM}$ MES/imidazole $\mathrm{pH}$ 6.5, 6\% ( $w / v)$ PEG 20K, 12\% ( $/ v$ ) PEG MME 550, 120 mM 1,6-hexanediol, $120 \mathrm{mM}$ 1-butanol, $120 \mathrm{mM}(R S)$-1,2-propanediol, $120 \mathrm{mM}$ 2-propanol and $120 \mathrm{mM}$

680 1,4-butanediol. Crystals were grown at room temperature for one week, subsequently harvested and flash-cooled in liquid nitrogen. Diffraction data were collected at an inhouse X-ray generator and processed, scaled using the XDS (Kabsch, 2010) package to a resolution of $1.7 \AA$. Initial phases were obtained by Molecular Replacement using PHASER and the structure of CIpC1NTD (PDB: 3WDB) (Vasudevan et al., 2013) as

685 starting model. The model was improved in iterative cycles of manual building using Coot (Emsley and Cowtan, 2004) and refinement with Phenix (Liebschner et al., 2019) omitting $5 \%$ of randomly selected reflections for calculation of $R_{\text {free. }}$ Model quality was monitored using MolProbity (Williams et al., 2018) and the final model exhibited good stereochemistry with $97.5 \%$ of residues in favored regions of the Ramachandran plot 690 and without any outliers. Structural illustrations were made using Pymol (Schrodinger, 2015).

\section{In vivo degradation assays}

A stationary culture of $M$. smegmatis $m^{2}-155$ carrying a plasmid for BRDT $\mathrm{BD} 1$ expression was diluted 1:200 in $50 \mathrm{~mL} 7 \mathrm{H} 9$ medium supplemented with $50 \mu \mathrm{g} / \mathrm{mL}$

695 hygromycin. The culture was grown at $37^{\circ} \mathrm{C}$ under vigorous shaking to an $\mathrm{OD} 600$ of $\sim 1$. Expression of BRDT $\mathrm{BD} 1$ was subsequently induced by addition of $200 \mu \mathrm{L} 50 \%(\mathrm{w} / \mathrm{v})$ acetamide in $\mathrm{H}_{2} \mathrm{O}$. The culture was maintained for additional 7 hours thereby reaching an $\mathrm{OD}_{600}$ of $\sim 4$. Cells were harvested by centrifugation and resuspended to an $\mathrm{OD}_{600}$ of 5 in fresh $7 \mathrm{H} 9$ medium. $270 \mu \mathrm{L}$ aliquots of the culture were transferred in a 96-well

700 glass-coated microplate (Thermo Scientific) and supplemented with either $1 \%(\mathrm{v} / \mathrm{v})$ DMSO, $100 \mu \mathrm{M}, 10 \mu \mathrm{M}$, or $1 \mu \mathrm{M}$ BacPROTAC-3, $100 \mu \mathrm{M}$ sCym-1, or $100 \mu \mathrm{M}$ JQ1 (Note, the medium of contained final $1 \%(v / v)$ DMSO). Experiments were performed 
in triplicates. Aliquots of $250 \mu \mathrm{L}$ of each cell suspension were withdrawn after 30 minutes incubation, cells were harvested, and the pellets were flash-frozen in liquid nitrogen. Cell pellets were thawed, resuspended in $100 \mu \mathrm{L}$ resuspension buffer (50 mM HEPES-NaOH pH 7.2, $150 \mathrm{mM} \mathrm{KCl}$ ) and lysed for 10 minutes using the Bioruptor (Diagenode, 10 cycles, 30 seconds on - 30 seconds off) in presence of small amounts of glass beads. Lysates were clarified by centrifugation, flash-frozen and stored at -80 ${ }^{\circ} \mathrm{C}$.

710 Cytosolic amounts of BRDT $T_{\mathrm{BD} 1}$ and $\mathrm{RpoB}$ in the lysates were quantified using Wes (ProteinSimple). Bacterial lysates were diluted 2-fold and analyzed using the Protein Simple 12-230 kDa Wes Separation Module following the manufacturer's instructions. Anti-BRDT (Bio-Vision) and anti-RpoB (BioLegend) antibodies were combined in a single primary antibody mixture for simultaneous $B R D T_{B D 1}$ and $\mathrm{RpoB}$ detection. The

715 primary antibody mixture contained anti-BRDT diluted 1:250 and anti-RpoB diluted 1:25,000 in Protein Simple Wes Antibody Diluent 2. BRDT BD1 $_{\text {and RpoB were detected }}$ using the Anti-Mouse (Protein Simple) and Anti-Rabbit (ProteinSimple) detection modules for Wes following the manufacturer's instruction. Anti-Mouse and anti-Rabbit antibodies were combined 1:9 and the obtained secondary antibody mixtures were 720 used for detection. Results were analyzed using the Compass for SW software (ProteinSimple). Compass displays the chemiluminescent signal detected along the length of the capillaries as electropherograms, where the intensity of the chemiluminescent signal is plotted against the apparent molecular weight (Figure S7). Detected peaks were quantified and include a peak at the expected BRDT BD1 $_{1}$ molecular weight $\left(B R D T_{\mathrm{BD} 1} \mathrm{MW}=16.6 \mathrm{kDa}\right.$ ) and an additional peak at the expected RpoB molecular weight $(\mathrm{MW}=128.5 \mathrm{kDa}$ ). Peak areas were normalized to DMSOtreated cells and plotted as mean \pm SD (Figure 5D and S7G).

\section{Sample preparation for quantitative mass spectrometry analysis}

For quantitative MS analysis, M. smegmatis cells expressing BRDT BD1 $_{\text {were }}$ treated 730 for 2 hours with $1 \%(v / v)$ DMSO, $100 \mu \mathrm{M}, 10 \mu \mathrm{M}$, or $1 \mu \mathrm{M}$ BacPROTAC-3, following the procedures described above. Cleared lysates were processed according to the single-pot SP3 protocol (Hughes et al., 2019) for low input proteomics sample preparation. Each lysate of $100 \mu \mathrm{l}$ was reduced with $10 \mathrm{mM}$ dithiothreitol (DTT, Sigma Aldrich) for 45 minutes at $37^{\circ} \mathrm{C}$ and subsequently alkylated with $20 \mathrm{mM}$ iodoacetamide 
735 (IAA, Sigma Aldrich) at room temperature for 1 hour. In parallel, a 1:1 mixture of 50 $\mathrm{mg} / \mathrm{mL}$ Sera-Mag SpeedBeads (GE Healthcare) and $50 \mathrm{mg} / \mathrm{mL}$ Sera-Mag SpeedBeads (GE Healthcare), exhibiting different surface hydrophilicity, was prepared in water. To each lysate $15 \mu \mathrm{L}$ of the prepared SP3 bead stock was added and binding was induced by the addition of $100 \mu \mathrm{L}$ ethanol. To ensure proper binding, samples

740 were incubated on a shaker for 5 minutes at $24^{\circ} \mathrm{C}$ and $1000 \mathrm{rpm}$. After protein binding, beads were washed 3 times with $200 \mu \mathrm{L}$ rinsing solution $(80 \%(v / v)$ ethanol in water) while being kept on a magnetic rack. Protein elution from the beads was enforced by addition of $100 \mathrm{mM}$ triethylammonium bicarbonate $(\mathrm{pH}=8.5$, Sigma Aldrich). To disaggregate the beads, the tubes were shortly sonicated in a water bath. For protein

745 digestion 1:25 wt/wt ratio of trypsin to protein was added and the samples were incubated overnight at $37^{\circ} \mathrm{C}$ in a thermo-shaker at $1,000 \mathrm{rpm}$.

Peptides were labelled for quantification in a multiplexed setup with TMT isobaric mass tags (TMTpro ${ }^{\mathrm{TM}}$ 16plex). Sample amount and quality was determined by HPLC-UV using a Dionex UltiMate 3000 HPLC RSLC nanosystem with a PepSwift Monolithic

750 RSLC column $\left(0.2 \times 5 \mathrm{~mm}\right.$, Thermo Fisher Scientific) at $60{ }^{\circ} \mathrm{C}$. Peptides were separated using a 20 minutes $2-90 \%$ elution gradient of buffer $A(80 \%(v / v) A C N, 0.1 \%$ (v/v) TFA in aqueous solution). For the labelling procedure, one TMTpro set was split into 3 aliquots to label 3 replicates. 14 channels (TMTpro 126-133N Da) were distributed over 2 timepoints for each of the 7 treatments. The 2 remaining channels

755 (133C and 134N) were used as reference channels with pools of all samples. Each sample was tagged with an excess of the respective TMT labelling reagent $(1: 20$, peptide:TMT label) and incubated at room temperature for 1 hour. The reaction was quenched by addition of $5 \mu \mathrm{L} 5 \%(\mathrm{v} / \mathrm{v})$ hydroxylamine (Sigma Aldrich), followed by a 15 minutes incubation step. For each replicate all 16 channels were pooled, and the

760 volume was reduced to $100 \mu \mathrm{L}$ in a speedvac. Removal of excess TMT labelling reagent was achieved by running the samples through tips filled with silica gel equilibrated in water.

\section{High pH chromatography and LC-MS/MS analysis}

Peptides were separated using a 40 min $2-50 \%$ gradient of buffer $\mathrm{A}$ in a high $\mathrm{pH}$ 765 chromatography (TEA, $\mathrm{pH}=8.5$ ) setup using a Dionex UltiMate 3000 HPLC RSLC nanosystem with a XBridge Peptide BEH C18 Column (1 x 150 mm, $130 \AA$ A, $3.5 \mu \mathrm{m}$, 
Waters). 40 fractions were collected and pooled by combining every $11^{\text {th }}$ fraction to generate a final number of 10 fractions for each replicate. The volume of each sample was adjusted to $100 \mu \mathrm{L}$ and the sample amount was estimated by running monolithic control runs.

LC-MS/MS analysis was performed on a Dionex UltiMate 3000 HPLC RSLC nanosystem using an Acclaim PepMap C-18 precolumn (0.3 x $5 \mathrm{~mm}$, Thermo Fisher Scientific) and an Acclaim PepMap C-18 column (50 cm x $75 \mu \mathrm{m}$, Thermo Fisher Scientific) coupled to a $Q$ Exactive HF-X hybrid quadrupole-Orbitrap mass spectrometer (Thermo Fisher Scientific). Peptides were separated using a 120 min linear gradient of $2-35 \%$ buffer $A$ at a flowrate of $230 \mathrm{~nL} / \mathrm{min}$. MS1 spectra were generated in a 380-1,650 m/z mass range at a 120,000 orbitrap resolution, AGC target of $3 \mathrm{e} 6$, with a maximum injection time of $50 \mathrm{~ms}$. The top 10 precursors were selected for MS2 analysis using a $0.7 \mathrm{~m} / \mathrm{z}$ quadrupole precursor isolation window, allowing

780 charge states 2-4 and a dynamic precursor exclusion of $30 \mathrm{~s}$. The orbitrap was operated at 45,000 resolution with an AGC of 1 e5 and a NCE of 35 at a maximum injection time of $250 \mathrm{~ms}$.

\section{MS data analysis}

MS raw data were analyzed using Proteome Discoverer 2.3 (PD 2.3.0.523, Thermo)

785 and the search was performed using the search engine MS Amanda (Dorfer et al., 2014) against a database of the M. smegmatis 2019 Uniprot Reference Proteome with contaminants and the BRDT $\mathrm{BD}_{\mathrm{B}}$ protein added. The database search allowed tryptic peptides with two missed cleavages at a precursor mass tolerance of 5 ppm and 0.02 Da MS2 tolerance. Static alkylation of cysteine and variable oxidation of methionine

790 and TMTpro adducts on lysine and peptide $\mathrm{N}$-termini were considered. Peptides were scored and filtered using Percolator (Kall et al., 2007) to obtain peptides at a $1 \%$ false discovery rate. Reporter ions were quantified using the IMP Hyperplex (https://ms.imp.ac.at/?goto=pd-nodes) at a reporter mass tolerance of $10 \mathrm{ppm}$ with a MS2 precursor threshold of 10. The search was performed for each replicate 795 separately over the 10 fractions.

Statistical analysis and data normalization were performed in R. The samples were first normalized for different sample loading by their total sum within each replicate set and then the three TMT replicates were normalized using the Internal Reference 
Scaling (IRS) method (Plubell et al., 2017). Median alignment was done afterwards by 800 TMM normalization. For each protein, the fold change of TMT-intensities and the corresponding $\mathrm{P}$ value (two-tailed Student's T-test,) were calculated. Permutationbased FDR calculation was used to assess the q-values. The mass spectrometry proteomics data have been deposited to the ProteomeXchange Consortium via the PRIDE (Perez-Riverol et al., 2019) partner repository with the dataset identifier 805 PXD021505.

\section{Data availability}

Coordinates of the ClpC NTD:SCym-1 crystal structure have been deposited at the Protein Data Bank (PDBe) under accession code 7AA4. Cryo-EM maps and atomic coordinates have been deposited in the EMDB and PDB with accession codes EMD-

81011708 for the ClpC tetramer-of-hexamers; EMD-11707 and PDB 7ABR for the single $\mathrm{ClpC}$ hexamer composing the assembly. The raw micrographs were submitted to the EMPIAR database (deposition ID: 847). The mass spectrometry proteomics data have been deposited to the ProteomeXchange Consortium via the PRIDE partner repository with the dataset identifier PXD021505. 


\section{REFERENCES}

Afonine, P.V., Poon, B.K., Read, R.J., Sobolev, O.V., Terwilliger, T.C., Urzhumtsev, A., and Adams, P.D. (2018). Real-space refinement in PHENIX for cryo-EM and crystallography. Acta Crystallogr D Struct Biol 74, 531-544.

820 Banik, S.M., Pedram, K., Wisnovsky, S., Ahn, G., Riley, N.M., and Bertozzi, C.R. (2020). Lysosome-targeting chimaeras for degradation of extracellular proteins. Nature 584, 291-297.

Barad, B.A., Echols, N., Wang, R.Y., Cheng, Y., DiMaio, F., Adams, P.D., and Fraser, J.S. (2015). EMRinger: side chain-directed model and map validation for 3D cryo825 electron microscopy. Nat Methods 12, 943-946.

Barbie, P., and Kazmaier, U. (2016). Total Synthesis of Cyclomarin A, a Marine Cycloheptapeptide with Anti-Tuberculosis and Anti-Malaria Activity. Org Lett 18, 204207.

Burslem, G.M., and Crews, C.M. (2020). Proteolysis-Targeting Chimeras as

830 Therapeutics and Tools for Biological Discovery. Cell 181, 102-114.

Carroni, M., Franke, K.B., Maurer, M., Jager, J., Hantke, I., Gloge, F., Linder, D., Gremer, S., Turgay, K., Bukau, B., et al. (2017). Regulatory coiled-coil domains promote head-to-head assemblies of AAA+ chaperones essential for tunable activity control. Elife 6.

835 Churcher, I. (2018). Protac-Induced Protein Degradation in Drug Discovery: Breaking the Rules or Just Making New Ones? J Med Chem 61, 444-452.

DeJesus, M.A., Gerrick, E.R., Xu, W., Park, S.W., Long, J.E., Boutte, C.C., Rubin, E.J., Schnappinger, D., Ehrt, S., Fortune, S.M., et al. (2017). Comprehensive Essentiality Analysis of the Mycobacterium tuberculosis Genome via Saturating 840 Transposon Mutagenesis. mBio 8.

Demonte, D., Drake, E.J., Lim, K.H., Gulick, A.M., and Park, S. (2013). Structurebased engineering of streptavidin monomer with a reduced biotin dissociation rate. Proteins 81, 1621-1633.

Deville, C., Franke, K., Mogk, A., Bukau, B., and Saibil, H.R. (2019). Two-Step 845 Activation Mechanism of the ClpB Disaggregase for Sequential Substrate Threading by the Main ATPase Motor. Cell Rep 27, 3433-3446 e3434. 
Dorfer, V., Pichler, P., Stranzl, T., Stadlmann, J., Taus, T., Winkler, S., and Mechtler, K. (2014). MS Amanda, a universal identification algorithm optimized for high accuracy tandem mass spectra. J Proteome Res 13, 3679-3684.

850 Emsley, P., and Cowtan, K. (2004). Coot: model-building tools for molecular graphics. Acta Crystallogr D Biol Crystallogr 60, 2126-2132.

Emsley, P., Lohkamp, B., Scott, W.G., and Cowtan, K. (2010). Features and development of Coot. Acta Crystallogr D Biol Crystallogr 66, 486-501.

Filippakopoulos, P., Picaud, S., Mangos, M., Keates, T., Lambert, J.P., Barsyte-

855 Lovejoy, D., Felletar, I., Volkmer, R., Muller, S., Pawson, T., et al. (2012). Histone recognition and large-scale structural analysis of the human bromodomain family. Cell 149, 214-231.

Gates, S.N., Yokom, A.L., Lin, J., Jackrel, M.E., Rizo, A.N., Kendsersky, N.M., Buell, C.E., Sweeny, E.A., Mack, K.L., Chuang, E., et al. (2017). Ratchet-like polypeptide 860 translocation mechanism of the AAA+ disaggregase Hsp104. Science 357, 273-279.

Goddard, T.D., Huang, C.C., and Ferrin, T.E. (2007). Visualizing density maps with UCSF Chimera. J Struct Biol 157, 281-287.

Goddard, T.D., Huang, C.C., Meng, E.C., Pettersen, E.F., Couch, G.S., Morris, J.H., and Ferrin, T.E. (2018). UCSF ChimeraX: Meeting modern challenges in visualization 865 and analysis. Protein Sci 27, 14-25.

Hanzl, A., and Winter, G.E. (2020). Targeted protein degradation: current and future challenges. Curr Opin Chem Biol 56, 35-41.

Hughes, C.S., Moggridge, S., Muller, T., Sorensen, P.H., Morin, G.B., and Krijgsveld, J. (2019). Single-pot, solid-phase-enhanced sample preparation for proteomics 870 experiments. Nat Protoc 14, 68-85.

Inobe, T., Fishbain, S., Prakash, S., and Matouschek, A. (2011). Defining the geometry of the two-component proteasome degron. Nat Chem Biol 7, 161-167.

Kabsch, W. (2010). Xds. Acta Crystallogr D Biol Crystallogr 66, 125-132.

Kall, L., Canterbury, J.D., Weston, J., Noble, W.S., and MacCoss, M.J. (2007). Semi875 supervised learning for peptide identification from shotgun proteomics datasets. Nat Methods 4, 923-925. 
Lee, H., and Suh, J.W. (2016). Anti-tuberculosis lead molecules from natural products targeting Mycobacterium tuberculosis ClpC1. J Ind Microbiol Biotechnol 43, 205-212. Leodolter, J., Warweg, J., and Weber-Ban, E. (2015). The Mycobacterium tuberculosis 880 ClpP1P2 Protease Interacts Asymmetrically with Its ATPase Partners ClpX and ClpC1. PLoS One 10, e0125345.

Lewis, K. (2020). The Science of Antibiotic Discovery. Cell 181, 29-45.

Li, Z., Wang, C., Wang, Z., Zhu, C., Li, J., Sha, T., Ma, L., Gao, C., Yang, Y., Sun, Y., et al. (2019). Allele-selective lowering of mutant HTT protein by HTT-LC3 linker 885 compounds. Nature 575, 203-209.

Liebschner, D., Afonine, P.V., Baker, M.L., Bunkoczi, G., Chen, V.B., Croll, T.I., Hintze, B., Hung, L.W., Jain, S., McCoy, A.J., et al. (2019). Macromolecular structure determination using $\mathrm{X}$-rays, neutrons and electrons: recent developments in Phenix. Acta Crystallogr D Struct Biol 75, 861-877.

890 Lim, K.H., Huang, H., Pralle, A., and Park, S. (2013). Stable, high-affinity streptavidin monomer for protein labeling and monovalent biotin detection. Biotechnol Bioeng 110, 57-67.

Liu, J., Mei, Z., Li, N., Qi, Y., Xu, Y., Shi, Y., Wang, F., Lei, J., and Gao, N. (2013). Structural dynamics of the MecA-ClpC complex: a type II AAA+ protein unfolding machine. J Biol Chem 288, 17597-17608.

Lopez, K.E., Rizo, A.N., Tse, E., Lin, J., Scull, N.W., Thwin, A.C., Lucius, A.L., Shorter, J., and Southworth, D.R. (2020). Conformational plasticity of the ClpAP AAA+ protease couples protein unfolding and proteolysis. Nat Struct Mol Biol 27, 406-416.

Matzuk, M.M., McKeown, M.R., Filippakopoulos, P., Li, Q., Ma, L., Agno, J.E., 900 Lemieux, M.E., Picaud, S., Yu, R.N., Qi, J., et al. (2012). Small-molecule inhibition of BRDT for male contraception. Cell 150, 673-684.

Maurer, M., Linder, D., Franke, K.B., Jager, J., Taylor, G., Gloge, F., Gremer, S., Le Breton, L., Mayer, M.P., Weber-Ban, E., et al. (2019). Toxic Activation of an AAA+ Protease by the Antibacterial Drug Cyclomarin A. Cell Chem Biol 26, 1169-1179 905 e1164. 
Neklesa, T.K., Tae, H.S., Schneekloth, A.R., Stulberg, M.J., Corson, T.W., Sundberg, T.B., Raina, K., Holley, S.A., and Crews, C.M. (2011). Small-molecule hydrophobic tagging-induced degradation of HaloTag fusion proteins. Nat Chem Biol 7, 538-543.

Nishimura, K., Fukagawa, T., Takisawa, H., Kakimoto, T., and Kanemaki, M. (2009).

910 An auxin-based degron system for the rapid depletion of proteins in nonplant cells. Nat Methods 6, 917-922.

Payne, D.J., Gwynn, M.N., Holmes, D.J., and Pompliano, D.L. (2007). Drugs for bad bugs: confronting the challenges of antibacterial discovery. Nat Rev Drug Discov 6, 29-40.

915 Perez-Riverol, Y., Csordas, A., Bai, J., Bernal-Llinares, M., Hewapathirana, S., Kundu, D.J., Inuganti, A., Griss, J., Mayer, G., Eisenacher, M., et al. (2019). The PRIDE database and related tools and resources in 2019: improving support for quantification data. Nucleic Acids Res 47, D442-D450.

Plubell, D.L., Wilmarth, P.A., Zhao, Y., Fenton, A.M., Minnier, J., Reddy, A.P., Klimek, 920 J., Yang, X., David, L.L., and Pamir, N. (2017). Extended Multiplexing of Tandem Mass Tags (TMT) Labeling Reveals Age and High Fat Diet Specific Proteome Changes in Mouse Epididymal Adipose Tissue. Mol Cell Proteomics 16, 873-890.

Prakash, S., Tian, L., Ratliff, K.S., Lehotzky, R.E., and Matouschek, A. (2004). An unstructured initiation site is required for efficient proteasome-mediated degradation.

$925 \quad$ Nat Struct Mol Biol 11, 830-837.

Puchades, C., Sandate, C.R., and Lander, G.C. (2020). The molecular principles governing the activity and functional diversity of AAA+ proteins. Nat Rev Mol Cell Biol 21, 43-58.

Punjani, A., Rubinstein, J.L., Fleet, D.J., and Brubaker, M.A. (2017). cryoSPARC: 930 algorithms for rapid unsupervised cryo-EM structure determination. Nat Methods 14, 290-296.

Rengarajan, J., Bloom, B.R., and Rubin, E.J. (2005). Genome-wide requirements for Mycobacterium tuberculosis adaptation and survival in macrophages. Proc Natl Acad Sci U S A 102, 8327-8332. 
935 Rizo, A.N., Lin, J., Gates, S.N., Tse, E., Bart, S.M., Castellano, L.M., DiMaio, F., Shorter, J., and Southworth, D.R. (2019). Structural basis for substrate gripping and translocation by the ClpB AAA+ disaggregase. Nat Commun 10, 2393.

Sakamoto, K.M., Kim, K.B., Kumagai, A., Mercurio, F., Crews, C.M., and Deshaies, R.J. (2001). Protacs: chimeric molecules that target proteins to the Skp1-Cullin-F box 940 complex for ubiquitination and degradation. Proc Natl Acad Sci U S A 98, 8554-8559.

Schapira, M., Calabrese, M.F., Bullock, A.N., and Crews, C.M. (2019). Targeted protein degradation: expanding the toolbox. Nat Rev Drug Discov 18, 949-963.

Schlothauer, T., Mogk, A., Dougan, D.A., Bukau, B., and Turgay, K. (2003). MecA, an adaptor protein necessary for ClpC chaperone activity. Proc Natl Acad Sci U S A 100, 2306-2311.

Schmidt, A., Trentini, D.B., Spiess, S., Fuhrmann, J., Ammerer, G., Mechtler, K., and Clausen, T. (2014). Quantitative phosphoproteomics reveals the role of protein arginine phosphorylation in the bacterial stress response. Mol Cell Proteomics 13, 537-550.

950 Schmitt, E.K., Riwanto, M., Sambandamurthy, V., Roggo, S., Miault, C., Zwingelstein, C., Krastel, P., Noble, C., Beer, D., Rao, S.P., et al. (2011). The natural product cyclomarin kills Mycobacterium tuberculosis by targeting the $\mathrm{ClpC} 1$ subunit of the caseinolytic protease. Angew Chem Int Ed Engl 50, 5889-5891.

Schrodinger, LLC (2015). The PyMOL Molecular Graphics System, Version 1.8.

955 Takahashi, D., Moriyama, J., Nakamura, T., Miki, E., Takahashi, E., Sato, A., Akaike, T., Itto-Nakama, K., and Arimoto, H. (2019). AUTACs: Cargo-Specific Degraders Using Selective Autophagy. Mol Cell 76, 797-810 e710.

Tommasi, R., Brown, D.G., Walkup, G.K., Manchester, J.I., and Miller, A.A. (2015). ESKAPEing the labyrinth of antibacterial discovery. Nat Rev Drug Discov 14, 529-542.

960 Trentini, D.B., Suskiewicz, M.J., Heuck, A., Kurzbauer, R., Deszcz, L., Mechtler, K., and Clausen, T. (2016). Arginine phosphorylation marks proteins for degradation by a Clp protease. Nature 539, 48-53.

Vasudevan, D., Rao, S.P., and Noble, C.G. (2013). Structural basis of mycobacterial inhibition by cyclomarin A. J Biol Chem 288, 30883-30891. 
965 Wagner, T., Merino, F., Stabrin, M., Moriya, T., Antoni, C., Apelbaum, A., Hagel, P., Sitsel, O., Raisch, T., Prumbaum, D., et al. (2019). SPHIRE-crYOLO is a fast and accurate fully automated particle picker for cryo-EM. Commun Biol 2, 218.

Wang, F., Mei, Z., Qi, Y., Yan, C., Hu, Q., Wang, J., and Shi, Y. (2011). Structure and mechanism of the hexameric MecA-ClpC molecular machine. Nature 471, 331-335.

970 Williams, C.J., Headd, J.J., Moriarty, N.W., Prisant, M.G., Videau, L.L., Deis, L.N., Verma, V., Keedy, D.A., Hintze, B.J., Chen, V.B., et al. (2018). MolProbity: More and better reference data for improved all-atom structure validation. Protein Sci 27, 293315.

Winter, G.E., Buckley, D.L., Paulk, J., Roberts, J.M., Souza, A., Dhe-Paganon, S., and 975 Bradner, J.E. (2015). DRUG DEVELOPMENT. Phthalimide conjugation as a strategy for in vivo target protein degradation. Science 348, 1376-1381.

World Health Organization (2019). Global tuerculosis report W.H.O. (WHO), ed. (Geneva, Switzerland).

Zengerle, M., Chan, K.H., and Ciulli, A. (2015). Selective Small Molecule Induced 980 Degradation of the BET Bromodomain Protein BRD4. ACS Chem Biol 10, 1770-1777. Zhang, K. (2016). Gctf: Real-time CTF determination and correction. J Struct Biol 193, 1-12.

Zheng, S.Q., Palovcak, E., Armache, J.P., Verba, K.A., Cheng, Y., and Agard, D.A. (2017). MotionCor2: anisotropic correction of beam-induced motion for improved cryoelectron microscopy. Nat Methods 14, 331-332.

Zivanov, J., Nakane, T., Forsberg, B.O., Kimanius, D., Hagen, W.J., Lindahl, E., and Scheres, S.H. (2018). New tools for automated high-resolution cryo-EM structure determination in RELION-3. Elife 7. 